\title{
Optimal shrinkage covariance matrix estimation under random sampling from elliptical distributions
}

\author{
Esa Ollila, Member, IEEE, and Elias Raninen, Student Member, IEEE
}

\begin{abstract}
This paper considers the problem of estimating a high-dimensional (HD) covariance matrix when the sample size is smaller, or not much larger, than the dimensionality of the data, which could potentially be very large. We develop a regularized sample covariance matrix (RSCM) estimator which can be applied in commonly occurring sparse data problems. The proposed RSCM estimator is based on estimators of the unknown optimal (oracle) shrinkage parameters that yield the minimum mean squared error (MMSE) between the RSCM and the true covariance matrix when the data is sampled from an unspecified elliptically symmetric distribution. We propose two variants of the RSCM estimator which differ in the approach in which they estimate the underlying sphericity parameter involved in the theoretical optimal shrinkage parameter. The performance of the proposed RSCM estimators are evaluated with numerical simulation studies. In particular when the sample sizes are low, the proposed RSCM estimators often show a significant improvement over the conventional RSCM estimator by Ledoit and Wolf (2004). We further evaluate the performance of the proposed estimators in classification and portfolio optimization problems with real data wherein the proposed methods are able to outperform the benchmark methods.
\end{abstract}

Index Terms-Sample covariance matrix, shrinkage estimation, regularization, elliptical distribution

\section{INTRODUCTION}

$\mathbf{E}$ STIMATING high-dimensional covariance matrices where the sample size $n$ is smaller, or not much larger than the dimension $p$ of the samples, is a problem that has attracted significant research interest in the recent years [1] [9]. This is due to the fact that high-dimensional data analysis problems have become increasingly common in a wide spectrum of fields, such as in finance [2], bioinformatics, and classification [10].

We consider the problem of estimating the high-dimensional covariance matrix based on a sample $\mathbf{x}_{1}, \ldots, \mathbf{x}_{n}$ of independent and identically distributed (i.i.d.) random vectors. The observations are assumed to be generated from an unspecified $p$-variate distribution $\mathbf{x} \sim F$ with a mean vector $\boldsymbol{\mu}=\mathbb{E}[\mathbf{x}]$ and a $p \times p$ positive definite covariance matrix

$$
\boldsymbol{\Sigma}=\mathbb{E}\left[(\mathbf{x}-\boldsymbol{\mu})(\mathbf{x}-\boldsymbol{\mu})^{\top}\right] \in \mathbb{S}_{++}^{p \times p} .
$$

The most commonly used estimators of the unknown parameters $(\boldsymbol{\mu}, \boldsymbol{\Sigma}) \in \mathbb{R}^{p} \times \mathbb{S}_{++}^{p \times p}$ are the sample mean vector and the

E. Ollila and E. Raninen are with the Department of Signal Processing and Acoustics, Aalto University, Espoo, P.O. Box 13000, FIN-00076 Aalto; email: \{esa.ollila,elias.raninen\}@aalto.fi (see http://users.spa.aalto.fi/esollila/), The research was supported by the Academy of Finland grant no. 298118 which is gratefully acknowledged

Manuscript received XX/20XX sample covariance matrix (SCM),

$$
\begin{aligned}
& \overline{\mathbf{x}}=\frac{1}{n} \sum_{i=1}^{n} \mathbf{x}_{i}, \\
& \mathbf{S}=\frac{1}{n-1} \sum_{i=1}^{n}\left(\mathbf{x}_{i}-\overline{\mathbf{x}}\right)\left(\mathbf{x}_{i}-\overline{\mathbf{x}}\right)^{\top} .
\end{aligned}
$$

They have desirable properties, such as being the sufficient statistics and unbiased. However, the SCM does not perform well in high-dimensional problems for several reasons. Foremost, significant estimation errors result from having an insufficient number of samples. Moreover, if $p>n$, the SCM is always singular, i.e., it is not invertible even though the true covariance matrix is known to be positive definite and hence non-singular. In these situations, a frequently used approach for improving the estimation accuracy is to use shrinkage regularization.

One of the most commonly used estimators in low sample support problems, where $p$ is large compared to the sample size $n$, is the regularized SCM (RSCM) of the form

$$
\mathbf{S}_{\alpha, \beta}=\beta \mathbf{S}+\alpha \mathbf{I},
$$

where $\alpha, \beta>0$ denote the shrinkage parameters or regularization parameters. In signal processing, an estimator of the form (1) was proposed in [11], [12] and is often referred to as the diagonal loading estimator. Another line of research has been to consider robust regularized covariance matrix estimators, e.g., [2]-[8], and [9]. In this paper, the focus is on determining the optimal (in MSE sense) shrinkage parameters for the RSCM.

We define the optimal RSCM estimator as the one that is based on the oracle shrinkage parameters minimizing the mean squared error (MSE), that is,

$$
\left(\alpha_{o}, \beta_{o}\right)=\underset{\alpha, \beta>0}{\arg \min } \mathbb{E}\left[\left\|\mathbf{S}_{\alpha, \beta}-\boldsymbol{\Sigma}\right\|_{\mathrm{F}}^{2}\right],
$$

where $\|\cdot\|_{\mathrm{F}}$ denotes the Frobenius matrix norm, i.e., $\|\mathbf{A}\|_{\mathrm{F}}^{2}=$ $\operatorname{tr}\left(\mathbf{A}^{\top} \mathbf{A}\right)=\operatorname{tr}\left(\mathbf{A} \mathbf{A}^{\top}\right)$ for any matrix $\mathbf{A}$. We use the prefix oracle for the shrinkage parameters $\left(\alpha_{o}, \beta_{o}\right)$ as they depend on the true unknown covariance matrix $\boldsymbol{\Sigma}$. Although, the oracle shrinkage parameters cannot be used in practice, they have the theoretical significance for being a benchmark for best possible performance w.r.t. the MSE metric.

The widely popular Ledoit-Wolf (LW-)RSCM [2] estimator is based on the consistent estimators $\left(\hat{\alpha}_{o}^{\mathrm{LW}}, \hat{\beta}_{o}^{\mathrm{LW}}\right)$ of the oracle parameters $\left(\alpha_{o}, \beta_{o}\right)$ under the random matrix theory (RMT) regime, i.e., as $n, p \rightarrow \infty$, we have

$$
c=p / n \rightarrow c_{0},
$$


It is, however, possible to improve upon the LW-estimator and obtain a more accurate finite sample estimation performance by assuming that the observations are generated from a specific $p$-variate distribution, e.g., the multivariate normal (MVN) distribution. For example, in [7, Theorem 1], the authors derived an optimal shrinkage parameter assuming that the samples have a Gaussian distribution with a known location $(\boldsymbol{\mu})$. Such a strict assumption on distribution of the data however implies performance loss if the assumption does not hold. Another somewhat related approach has been taken for example in [13, Proposition 3], where the authors considered robust $M$-estimators and looked for an asymptotically optimal shrinkage parameter in the RMT regime which minimizes the squared Frobenius distance between normalized regularized $M$-estimators of scatter matrix and a normalized covariance matrix.

In this paper, we instead assume that the observations are from an unspecified elliptically symmetric (ES) distribution and derive estimators of the optimal oracle shrinkage parameters $\left(\alpha_{o}, \beta_{o}\right)$ that are able to perform reliably under the RMT regime. ES distributions is a large class of distributions comprising, e.g., the MVN distribution, generalized Gaussian, and all compound Gaussian distributions as special cases, see e.g., [14], [15], and [16].

The rest of this paper is organized as follows. In Section II and Section III, we derive the optimal shrinkage parameters $\left(\alpha_{o}, \beta_{o}\right)$ under the general assumption of sampling from any $p$-variate distribution and an elliptical distribution with finite fourth order moments, respectively. In Section IV, we develop estimators of $\left(\alpha_{o}, \beta_{o}\right)$ under the RMT regime and when sampling from an unspecified elliptically symmetric distribution. In Section $\mathrm{V}$, we conduct several simulation studies and compare the proposed estimators with the popular LW-RSCM estimator. In Section VI, we illustrate the performance of the proposed estimators in two applications. First, the proposed methods are used in a regularized discriminant analysis (RDA) framework, where they are applied to the classification of phoneme data. Then the methods are used in a portfolio optimization problem using the Global Minimum Variance Portfolio (GMVP) framework, where we use real data of historical (daily) stock returns from the Hong Kong's Hang Seng Index (HSI) and Standard and Poor's 500 (SP500) index. In both applications the proposed methods are shown to perform better than the benchmark methods. Finally, Section VII concludes.

Notation: We denote the open cone of $p \times p$ positive definite symmetric matrices by $\mathbb{S}_{++}^{p \times p}$. The vectorization of an $n \times p$ matrix $\mathbf{A}=\left(\mathbf{a}_{1}, \ldots \mathbf{a}_{p}\right)$ is denoted by $\operatorname{vec}(\mathbf{A})=$ $\left(\mathbf{a}_{1}^{\top}, \ldots, \mathbf{a}_{p}^{\top}\right)^{\top}$. The matrix trace of a square matrix $\mathbf{A}$ is denoted by $\operatorname{tr}(\mathbf{A})$. The Kronecker product $\mathbf{A} \otimes \mathbf{B}$ of any matrices $\mathbf{A}$ and $\mathbf{B}$ is a block matrix with its $i j$ th block being equal to $a_{i j} \mathbf{B}$. Kronecker product has the useful property: $(\mathbf{A} \otimes \mathbf{B})(\mathbf{C} \otimes \mathbf{D})=(\mathbf{A C} \otimes \mathbf{B D})$ for the matrices $\mathbf{A}, \mathbf{B}$, $\mathbf{C}$, and $\mathbf{D}$ of appropriate dimensions. We denote the identity matrix of proper dimension by $\mathbf{I}$ and the centering matrix of

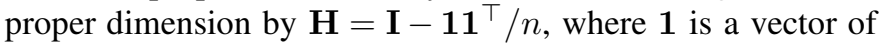
ones. The canonical basis vector, which has its $i$ th element equal to 1 and all other elements zero is denoted by $\mathbf{e}_{i}$. The commutation matrix $\mathbf{K}_{p}$ is a $p^{2} \times p^{2}$ block matrix with its $i j$ th block equal to a $p \times p$ matrix that has a 1 at element $j i$ and zeros elsewhere, i.e., $\mathbf{K}_{p}=\sum_{i, j} \mathbf{e}_{i} \mathbf{e}_{j}^{\top} \otimes \mathbf{e}_{j} \mathbf{e}_{i}^{\top}$. It also has the following important properties [17]: $\mathbf{K}_{p} \operatorname{vec}(\mathbf{A})=\operatorname{vec}\left(\mathbf{A}^{\top}\right)$ and $\mathbf{K}_{p}(\mathbf{A} \otimes \mathbf{B}) \mathbf{K}_{p}=(\mathbf{B} \otimes \mathbf{A})$ for any $p \times p$ matrices $\mathbf{A}$ and $\mathbf{B}$. In our developments, we will also use the following identities: $\operatorname{tr}(\mathbf{A} \otimes \mathbf{B})=\operatorname{tr}(\mathbf{A}) \operatorname{tr}(\mathbf{B}), \operatorname{tr}\left(\operatorname{vec}(\mathbf{A}) \operatorname{vec}(\mathbf{B})^{\top}\right)=$ $\operatorname{tr}\left(\mathbf{A}^{\top} \mathbf{B}\right)=\operatorname{vec}(\mathbf{B})^{\top} \operatorname{vec}(\mathbf{A})$ for any square matrices $\mathbf{A}$ and $\mathbf{B}$ of same order. Notation " $={ }_{d}$ "reads "has the same distribution as".

\section{OPTIMAL ORACLE SHRINKAGE PARAMETERS}

In this section, we derive the oracle shrinkage parameters $\left(\alpha_{o}, \beta_{o}\right)$ for any $p$-variate distribution. First, we define the scale and sphericity parameters of $\boldsymbol{\Sigma} \in \mathbb{S}_{++}^{p \times p}$ as

$$
\eta=\frac{\operatorname{tr}(\boldsymbol{\Sigma})}{p} \quad \text { and } \quad \gamma=\frac{p \operatorname{tr}\left(\boldsymbol{\Sigma}^{2}\right)}{\operatorname{tr}(\boldsymbol{\Sigma})^{2}}
$$

Note that $\eta$ equals the mean of the eigenvalues of $\boldsymbol{\Sigma}$ whereas $\gamma$ is equal to the ratio of the mean of the squared eigenvalues relative to mean of eigenvalues squared. The sphericity $\gamma[1]$, [18] measures how close the covariance matrix is to a scaled identity matrix. Furthermore, the values for the sphericity are in the range $1 \leq \gamma \leq p$. This can be seen by applying the Cauchy-Schwartz inequality:

$$
\operatorname{tr}(\boldsymbol{\Sigma})^{2}=\left(\sum_{i=1}^{p} \lambda_{i} \cdot 1\right)^{2} \leq p \cdot \sum_{i=1}^{p} \lambda_{i}^{2}=p \operatorname{tr}\left(\boldsymbol{\Sigma}^{2}\right) .
$$

By dividing the right-hand side of the equation by the left-hand side, we have $\gamma \geq 1$ with equality if and only if $\boldsymbol{\Sigma}=c \mathbf{I}$ for some $c>0$. Furthermore, the upper bound $\gamma=p$ is achieved for rank one matrices, in which case $\Sigma$ has only one non-zero eigenvalue.

The scale and sphericity, $\eta$ and $\gamma$, are elemental in our developments. As is shown in Theorem 3, the optimal shrinkage parameter pair $\left(\alpha_{o}, \beta_{o}\right)$ for a given elliptical distribution depends on the true covariance matrix $\boldsymbol{\Sigma}$ only through $\eta$ and $\gamma$. Simple plug-in estimates of $\left(\alpha_{o}, \beta_{o}\right)$ can then be obtained by replacing $(\eta, \gamma)$ with their estimates. If the elliptical distribution is unknown an additional elliptical kurtosis parameter needs to be estimated.

The next theorem provides the expressions for the oracle shrinkage parameters in the case of sampling from an unspecified $p$-variate distribution with finite fourth order moments. Write $\operatorname{MSE}(\mathbf{S})=\mathbb{E}\left[\|\mathbf{S}-\mathbf{\Sigma}\|_{\mathrm{F}}^{2}\right]$ for the mean squared error (MSE) and

$$
\operatorname{NMSE}(\mathbf{S})=\frac{\mathbb{E}\left[\|\mathbf{S}-\boldsymbol{\Sigma}\|_{\mathrm{F}}^{2}\right]}{\|\boldsymbol{\Sigma}\|_{\mathrm{F}}^{2}}
$$

for the normalized MSE.

Theorem 1. Let $\mathrm{x}_{1}, \ldots, \mathbf{x}_{n}$ denote an i.i.d. random sample from any $p$-variate distribution with finite fourth order mo- 
ments, mean vector $\boldsymbol{\mu}$, and covariance matrix $\boldsymbol{\Sigma}$. Then, the oracle shrinkage parameters in (2) are

$$
\begin{aligned}
\beta_{o} & =\frac{p(\gamma-1) \eta^{2}}{\mathbb{E}\left[\operatorname{tr}\left(\mathbf{S}^{2}\right)\right]-p \eta^{2}} \\
& =\frac{(\gamma-1)}{(\gamma-1)+\gamma \cdot \operatorname{NMSE}(\mathbf{S})}
\end{aligned}
$$

and

$$
\alpha_{o}=\left(1-\beta_{o}\right) \eta
$$

where $\eta$ and $\gamma$ are defined in (3). Furthermore, the optimal $\beta_{o}$ is always in the range $[0,1)$ and the value of the MSE at the optimum is

$$
\operatorname{MSE}\left(\mathbf{S}_{\alpha_{o}, \beta_{o}}\right)=\left(1-\beta_{o}\right)\|\boldsymbol{\Sigma}-\eta \mathbf{I}\|_{\mathrm{F}}^{2} .
$$

Proof. It was shown in [2, Theorem 2.1] that

$$
\beta_{o}=\frac{\|\boldsymbol{\Sigma}-\eta \mathbf{I}\|_{\mathrm{F}}^{2}}{\|\boldsymbol{\Sigma}-\eta \mathbf{I}\|_{\mathrm{F}}^{2}+\operatorname{MSE}(\mathbf{S})}
$$

and $\alpha_{o}=\left(1-\beta_{o}\right) \eta$. Although, the result was shown assuming $\boldsymbol{\mu}=\mathbf{0}$ is known and for $\mathbf{S}_{0}=(1 / n) \sum_{i=1}^{n} \mathbf{x}_{i} \mathbf{x}_{i}^{\top}$, this result transfers to the non-centered case as the derivation only assumes that $\mathbb{E}\left[\mathbf{S}_{0}\right]=\boldsymbol{\Sigma}$ which applies to $\mathbf{S}$ as well. Note that, $\beta_{o}=1$ only if $\mathbf{S}=\mathbf{\Sigma}$, and thus $\operatorname{MSE}(\mathbf{S})=0$, which has zero probability when sampling from a continuous distribution. The form of $\beta_{o}$ in (8) therefore implies that $\beta_{o} \in[0,1)$. We now show that (8) can be expressed in the form (4).

First, we write

$$
\begin{aligned}
a_{1} & =\operatorname{MSE}(\mathbf{S})=\mathbb{E}\left[\|\mathbf{S}-\boldsymbol{\Sigma}\|_{\mathrm{F}}^{2}\right] \\
& =\mathbb{E}\left[\operatorname{tr}\left(\mathbf{S}^{2}\right)\right]-2 \mathbb{E}[\operatorname{tr}(\mathbf{S} \boldsymbol{\Sigma})]+\operatorname{tr}\left(\boldsymbol{\Sigma}^{2}\right) \\
& =\mathbb{E}\left[\operatorname{tr}\left(\mathbf{S}^{2}\right)\right]-\operatorname{tr}\left(\boldsymbol{\Sigma}^{2}\right),
\end{aligned}
$$

where we used that $\mathbb{E}[\operatorname{tr}(\mathbf{S} \boldsymbol{\Sigma})]=\operatorname{tr}(\mathbb{E}[\mathbf{S}] \boldsymbol{\Sigma})=\operatorname{tr}\left(\boldsymbol{\Sigma}^{2}\right)$. The numerator of $\beta_{o}$ in (8) is

$$
\begin{aligned}
a_{2} & =\|\boldsymbol{\Sigma}-\eta \mathbf{I}\|_{\mathrm{F}}^{2}=\operatorname{tr}\left(\boldsymbol{\Sigma}^{2}\right)-(1 / p) \operatorname{tr}(\boldsymbol{\Sigma})^{2} \\
& =p\left(\vartheta-\eta^{2}\right)=p(\gamma-1) \eta^{2},
\end{aligned}
$$

where we denote $\vartheta=\operatorname{tr}\left(\boldsymbol{\Sigma}^{2}\right) / p$. This shows that the denominator of $\beta_{o}$ is $a_{1}+a_{2}=\mathbb{E}\left[\operatorname{tr}\left(\mathbf{S}^{2}\right)\right]-(1 / p) \operatorname{tr}(\boldsymbol{\Sigma})^{2}=$ $\mathbb{E}\left[\operatorname{tr}\left(\mathbf{S}^{2}\right)\right]-p \eta^{2}$. These expressions for the numerator and the denominator of $\beta_{o}$ yield the assertion (4) for $\beta_{o}$. Substituting (10) into (8) and multiplying both the numerator and denominator by $1 /\left(p \eta^{2}\right)$ gives $(5)$.

Next, we derive the expression for the MSE of the RSCM $\mathbf{S}_{\alpha, \beta}$. By using the variance and bias decomposition of the MSE, we have

$$
\begin{aligned}
\operatorname{MSE}\left(\mathbf{S}_{\alpha, \beta}\right) & =\operatorname{tr}\left(\operatorname{var}\left(\operatorname{vec}\left(\mathbf{S}_{\alpha, \beta}\right)\right)\right)+\left\|\mathbb{E}\left[\mathbf{S}_{\alpha, \beta}\right]-\boldsymbol{\Sigma}\right\|_{\mathrm{F}}^{2} \\
& =\beta^{2} \operatorname{tr}(\operatorname{var}(\operatorname{vec}(\mathbf{S})))+\|\alpha \mathbf{I}-(1-\beta) \boldsymbol{\Sigma}\|_{\mathrm{F}}^{2} \\
& =\beta^{2} \operatorname{MSE}(\mathbf{S})+\|\alpha \mathbf{I}-(1-\beta) \boldsymbol{\Sigma}\|_{\mathrm{F}}^{2}
\end{aligned}
$$

We used the fact that from the unbiasedness of $\mathbf{S}$ it follows that $\operatorname{MSE}(\mathbf{S})=\operatorname{tr}(\operatorname{var}(\operatorname{vec}(\mathbf{S})))=a_{1}$. At the optimum, we have $\beta_{o} a_{1}=\left(1-\beta_{o}\right) a_{2}$, which can be seen from (8), and $\alpha_{o}=\left(1-\beta_{o}\right) \eta$. The MSE at the optimum is therefore

$$
\begin{aligned}
\operatorname{MSE}\left(\mathbf{S}_{\left(1-\beta_{o}\right) \eta, \beta_{o}}\right) & =\beta_{o}^{2} \operatorname{MSE}(\mathbf{S})+\left(1-\beta_{o}\right)^{2}\|\mathbf{\Sigma}-\eta \mathbf{I}\|_{\mathrm{F}}^{2} \\
& =\beta_{o}\left(1-\beta_{o}\right) a_{2}+\left(1-\beta_{o}\right)^{2} a_{2} \\
& =\left(1-\beta_{o}\right) a_{2},
\end{aligned}
$$

which concludes the proof.

Theorem 1 has important implications. First, since $\alpha_{o}=$ $\left(1-\beta_{o}\right) \eta$ is determined by the value of $\beta_{o} \in[0,1)$, the optimal RSCM can be expressed as

$$
\mathbf{S}_{\alpha_{o}, \beta_{o}}=\beta_{o} \mathbf{S}+\left(1-\beta_{o}\right) \eta \mathbf{I} .
$$

The scale $\eta$ can be estimated with

$$
\hat{\eta}=\frac{\operatorname{tr}(\mathbf{S})}{p},
$$

which is a consistent estimator both in the conventional (fixed $p$ ) and the RMT asymptotic regime. Therefore, the estimator of $\alpha_{o}$ is simply $\hat{\alpha}_{o}=\left(1-\hat{\beta}_{o}\right) \hat{\eta}$, and we can focus on finding an estimator $\hat{\beta}_{o}$ of $\beta_{o}$.

This is the approach also taken by Ledoit and Wolf [2] who develop an estimator $\hat{\beta}_{o}^{\mathrm{LW}}$ that converges to $\beta_{o}$ in (4) under the RMT regime (R1) and some mild technical assumptions when sampling from a distribution $\mathbf{x} \sim F$ with finite 8th order moments. The estimate of $\alpha_{o}$ is then $\hat{\alpha}_{o}^{\mathrm{LW}}=\left(1-\hat{\beta}_{o}^{\mathrm{LW}}\right) \hat{\eta}$. The RSCM based on the shrinkage parameter pair $\left(\hat{\alpha}_{o}^{\mathrm{LW}}, \hat{\beta}_{o}^{\mathrm{LW}}\right)$ of [2] is referred hereafter as the $L W-R S C M$ estimator.

\section{OPTIMAL ORACLE SHRINKAGE PARAMETERS: THE ELLIPTICAL CASE}

We now derive the optimal oracle shrinkage parameters for the case in which the data can be assumed elliptically distributed. For a review of elliptical distributions, see [14], [15], and [16].

The probability density function (p.d.f.) of an elliptically distributed random vector $\mathbf{x} \sim \mathcal{E}_{p}(\boldsymbol{\mu}, \boldsymbol{\Sigma}, g)$ is

$$
f(\mathbf{x})=C_{p, g}|\boldsymbol{\Sigma}|^{-1 / 2} g\left((\mathbf{x}-\boldsymbol{\mu})^{\top} \boldsymbol{\Sigma}^{-1}(\mathbf{x}-\boldsymbol{\mu})\right),
$$

where $\mathbb{E}[\mathbf{x}]=\boldsymbol{\mu}$ is the mean vector, $\boldsymbol{\Sigma}$ is the positive definite covariance matrix, $g:[0, \infty) \rightarrow[0, \infty)$ is the density generator, which is a fixed function that is independent of $\mathbf{x}, \boldsymbol{\mu}$ and $\boldsymbol{\Sigma}$, and $C_{p, g}$ is a normalizing constant ensuring that $f(\mathbf{x})$ integrates to 1 . Here, we let $g$ to be defined so that $\boldsymbol{\Sigma}$ represents the covariance matrix of $\mathbf{x}$, which means that $\int_{0}^{\infty} t^{p / 2} g(t) \mathrm{d} t=p$. The functional form of the density generator $g$ determines the elliptical distribution. For example, the multivariate normal (MVN) distribution, denoted $\mathbf{x} \sim \mathcal{N}_{p}(\boldsymbol{\mu}, \boldsymbol{\Sigma})$, is obtained when $g(t)=\exp (-t / 2)$. As in Theorem 1, we assume that the elliptical population possesses finite fourth order moments. Technically, this implies that

$$
\int_{0}^{\infty} t^{p / 2+1} g(t) \mathrm{d} t<\infty .
$$

For example, the MVN and the multivariate $t$-distribution with degrees of freedom $\nu>4$ all verify the above condition. 
The kurtosis of a random variable $x$ is defined as

$$
\operatorname{kurt}(x)=\frac{\mathbb{E}\left[(x-\mu)^{4}\right]}{\left(\mathbb{E}\left[(x-\mu)^{2}\right]\right)^{2}}-3,
$$

where $\mu=\mathbb{E}[x]$. The elliptical kurtosis parameter [14] $\kappa$ of a random vector $\mathbf{x}=\left(x_{1}, \ldots, x_{p}\right)^{\top} \sim \mathcal{E}_{p}(\boldsymbol{\mu}, \boldsymbol{\Sigma}, g)$ is defined as

$$
\kappa=\frac{\mathbb{E}\left[r^{4}\right]}{p(p+2)}-1=\frac{1}{3} \cdot \operatorname{kurt}\left(x_{i}\right),
$$

where $r$ is the generating variate or second order modular variate of the elliptical distribution, which is defined as the square-root of the quadratic form $r^{2}=(\mathbf{x}-\boldsymbol{\mu})^{\top} \boldsymbol{\Sigma}^{-1}(\mathbf{x}-$ $\boldsymbol{\mu})$. Above $\operatorname{kurt}\left(x_{i}\right)$ denotes the kurtosis of (any) marginal variable $x_{i}$. The elliptical kurtosis shares properties similar to the kurtosis of a real random variable. Especially, if $\mathbf{x} \sim$ $\mathcal{N}_{p}(\boldsymbol{\mu}, \boldsymbol{\Sigma})$, then $\kappa=0$. This is obvious since the marginal distributions are Gaussian and hence $\kappa=(1 / 3) \operatorname{kurt}\left(x_{i}\right)=0$. Another way to derive this is by noting that the quadratic form $r^{2}$ has a chi-squared distribution with $p$ degrees of freedom, i.e., $r^{2} \sim \chi_{p}^{2}$, and hence $\mathbb{E}\left[r^{4}\right]=p(p+2)$.

The importance of the elliptical kurtosis parameter $\kappa$ is due to the fact that the $p^{2} \times p^{2}$ covariance matrix of vec $(\mathbf{S})$ depends on the underlying elliptical distribution $g$ only through $\kappa$. This result is established in Theorem 2.

We will utilize the following matrix decomposition in our proofs. Let $\mathbf{X}=\left(\mathbf{x}_{1} \cdots \mathbf{x}_{n}\right)^{\top}$ denote the $n \times p$ data matrix with $i$ th transposed observation as its row vector. Then, the SCM can be written as

$$
\mathbf{S}=\frac{1}{n-1} \mathbf{X}^{\top} \mathbf{H} \mathbf{X}
$$

where $\mathbf{H}$ is the centering matrix.

Theorem 2. Let $\mathbf{x}_{1}, \ldots, \mathbf{x}_{n}$ denote an i.i.d. random sample from an elliptical distribution with finite fourth order moments, mean vector $\boldsymbol{\mu}$, and covariance matrix $\boldsymbol{\Sigma}$. Then,

$$
\begin{gathered}
\operatorname{var}(\operatorname{vec}(\mathbf{S}))= \\
\left(\frac{1}{n-1}+\frac{\kappa}{n}\right)\left(\mathbf{I}+\mathbf{K}_{p}\right)(\boldsymbol{\Sigma} \otimes \boldsymbol{\Sigma})+\frac{\kappa}{n} \operatorname{vec}(\boldsymbol{\Sigma}) \operatorname{vec}(\boldsymbol{\Sigma})^{\top} .
\end{gathered}
$$

Proof. For elliptically distributed observations $\left\{\mathbf{x}_{i}\right\}_{i=1}^{n} \stackrel{i i d}{\sim}$ $\mathcal{E}_{p}(\boldsymbol{\mu}, \boldsymbol{\Sigma}, g)$, we have the following stochastic decomposition $\mathbf{x}_{i}={ }_{d} \boldsymbol{\Sigma}^{1 / 2} \mathbf{z}_{i}+\boldsymbol{\mu}$, where $\mathbf{z}_{i} \sim \mathcal{E}_{p}(\mathbf{0}, \mathbf{I}, g)$. Let $\mathbf{Z}=$ $\left(\mathbf{z}_{1} \cdots \mathbf{z}_{n}\right)^{\top}$ denote the $n \times p$ data matrix collecting the random vectors $\mathbf{z}_{i}$ as its row vectors. Then, the stochastic decomposition implies that

$$
\mathbf{X}^{\top} \mathbf{H X}={ }_{d} \boldsymbol{\Sigma}^{1 / 2} \mathbf{Z}^{\top} \mathbf{H Z} \boldsymbol{\Sigma}^{1 / 2} .
$$

Hence,

$$
\begin{aligned}
& \operatorname{var}(\operatorname{vec}(\mathbf{S}))=\left(\operatorname{var}\left(\frac{1}{n-1} \operatorname{vec}\left(\boldsymbol{\Sigma}^{1 / 2} \mathbf{Z}^{\top} \mathbf{H} \mathbf{Z} \boldsymbol{\Sigma}^{1 / 2}\right)\right)\right) \\
& =\left(\boldsymbol{\Sigma}^{1 / 2} \otimes \boldsymbol{\Sigma}^{1 / 2}\right) \operatorname{var}\left(\frac{1}{n-1} \operatorname{vec}\left(\mathbf{Z}^{\top} \mathbf{H Z}\right)\right)\left(\boldsymbol{\Sigma}^{1 / 2} \otimes \boldsymbol{\Sigma}^{1 / 2}\right) .
\end{aligned}
$$

Since the matrix $\mathbf{Z}^{\top} \mathbf{H Z}$ is radially distributed, we can apply [19], which states

$\operatorname{var}\left(\frac{1}{n-1} \operatorname{vec}\left(\mathbf{Z}^{\top} \mathbf{H Z}\right)\right)=\tau_{1}\left(\mathbf{I}+\mathbf{K}_{p}\right)+\tau_{2} \operatorname{vec}(\mathbf{I}) \operatorname{vec}(\mathbf{I})^{\top}$, where the parameters $\tau_{1}$ and $\tau_{2}$ correspond to the variance of any off-diagonal element and the covariance of any two diagonal elements of the matrix $\frac{1}{n-1} \mathbf{Z}^{\top} \mathbf{H Z}$, respectively.

We will first derive the expression for $\tau_{1}$. For $q \neq r$, it holds that

$$
\begin{aligned}
(n-1)^{2} \tau_{1} & =\operatorname{var}\left(\left(\mathbf{Z} \mathbf{e}_{q}\right)^{\top} \mathbf{H}\left(\mathbf{Z} \mathbf{e}_{r}\right)\right) \\
& =\operatorname{var}\left(\operatorname{tr}\left(\mathbf{H}\left(\mathbf{Z} \mathbf{e}_{r}\right)\left(\mathbf{Z} \mathbf{e}_{q}\right)^{\top}\right)\right) \\
& =\operatorname{var}\left(\operatorname{vec}(\mathbf{H})^{\top} \operatorname{vec}\left(\left(\mathbf{Z} \mathbf{e}_{r}\right)\left(\mathbf{Z} \mathbf{e}_{q}\right)^{\top}\right)\right) \\
& =\operatorname{vec}(\mathbf{H})^{\top} \operatorname{var}\left(\operatorname{vec}\left(\left(\mathbf{Z} \mathbf{e}_{r}\right)\left(\mathbf{Z} \mathbf{e}_{q}\right)^{\top}\right)\right) \operatorname{vec}(\mathbf{H}) .
\end{aligned}
$$

Next we recall that $\mathbf{z}_{i} \sim \mathcal{E}_{p}(\mathbf{0}, \mathbf{I}, g)$ has a stochastic representation ( $c f$. [15, Theorem 2.9]) $\mathbf{z}_{i}={ }_{d} r_{i} \mathbf{u}_{i}$, where $r_{i}$ is the generating variate with a density $f(r)=C \cdot r^{p-1} g\left(r^{2}\right)$ (where $C$ is normalizing constant) and $\mathbf{u}_{i}=\left(u_{i 1}, u_{i 2}, \ldots, u_{i p}\right)^{\top}$ is uniformly distributed on the unit hypersphere $\mathcal{S}^{p-1}=$ $\left\{\mathbf{x} \in \mathbb{R}^{p}: \mathbf{x}^{\top} \mathbf{x}=1\right\}$ and $r_{i}$ is independent of $\mathbf{u}_{i}$. Using this stochastic representation for $\mathbf{z}_{i}$, we can write $\mathbf{Z e}_{q}=\left(r_{1} u_{1 q}, r_{2} u_{2 q}, \ldots, r_{n} u_{n q}\right)^{\top}$, The $k l$ th element of the $i j$ th block (i.e., the $i j k l$ th element) of the $n^{2} \times n^{2}$ matrix $\operatorname{var}\left(\operatorname{vec}\left(\left(\mathbf{Z e}_{r}\right)\left(\mathbf{Z e}_{q}\right)^{\top}\right)\right)$ can then be written as

$$
\begin{aligned}
& \operatorname{cov}\left(\left(\mathbf{Z} \mathbf{e}_{r}\right)_{k}\left(\mathbf{Z} \mathbf{e}_{q}\right)_{i},\left(\mathbf{Z} \mathbf{e}_{r}\right)_{l}\left(\mathbf{Z} \mathbf{e}_{q}\right)_{j}\right)= \\
& \mathbb{E}\left[r_{k} u_{k r} \cdot r_{i} u_{i q} \cdot r_{l} u_{l r} \cdot r_{j} u_{j q}\right] \\
& \quad-\mathbb{E}\left[r_{k} u_{k r} \cdot r_{i} u_{i q}\right] \mathbb{E}\left[r_{l} u_{l r} \cdot r_{j} u_{j q}\right] .
\end{aligned}
$$

Using the following identities for $\forall i, j$ and $q \neq r$ ( $c f$. [15, Section 3.1]) :

$$
\begin{aligned}
\mathbb{E}\left[u_{i q} u_{j r}\right] & =0, & \mathbb{E}\left[u_{i q}^{2} u_{i r}^{2}\right] & =\frac{1}{p(p+2)}, \\
\mathbb{E}\left[u_{i q}^{2}\right] & =\frac{1}{p}, & \mathbb{E}\left[u_{i q}^{4}\right] & =\frac{3}{p(p+2)}, \\
\mathbb{E}\left[r_{i}^{2}\right] & =p, & \mathbb{E}\left[r_{i}^{4}\right] & =(1+\kappa) p(p+2),
\end{aligned}
$$

where $3 \kappa=\operatorname{kurt}\left(z_{i q}\right)=\operatorname{kurt}\left(x_{i q}\right)$, we find that the only nonzero elements of $\operatorname{var}\left(\operatorname{vec}\left(\left(\mathbf{Z e}_{r}\right)\left(\mathbf{Z e}_{q}\right)^{\top}\right)\right)$ correspond to

$$
\begin{aligned}
\mathbb{E}\left[r_{i}^{4}\right] \mathbb{E}\left[u_{i r}^{2} u_{i q}^{2}\right] & =1+\kappa & & \text { for } i=j=k=l, \text { and } \\
\mathbb{E}\left[r_{i}^{2}\right] \mathbb{E}\left[r_{k}^{2}\right] \mathbb{E}\left[u_{i r}^{2}\right] \mathbb{E}\left[u_{k q}^{2}\right] & =1 & & \text { for } i=j, k=l, i \neq k .
\end{aligned}
$$

This implies that

$$
\operatorname{var}\left(\operatorname{vec}\left(\left(\mathbf{Z} \mathbf{e}_{r}\right)\left(\mathbf{Z} \mathbf{e}_{q}\right)^{\top}\right)\right)=\mathbf{I}+\kappa \sum_{i=1}^{n} \mathbf{e}_{i} \mathbf{e}_{i}^{\top} \otimes \mathbf{e}_{i} \mathbf{e}_{i}^{\top} .
$$

Hence, we can write $\tau_{1}$ as

$$
\begin{aligned}
\tau_{1} & =\frac{1}{(n-1)^{2}} \operatorname{vec}(\mathbf{H})^{\top}\left(\mathbf{I}+\kappa \sum_{i=1}^{n} \mathbf{e}_{i} \mathbf{e}_{i}^{\top} \otimes \mathbf{e}_{i} \mathbf{e}_{i}^{\top}\right) \operatorname{vec}( \\
& =\frac{1}{n-1}+\frac{\kappa}{n}
\end{aligned}
$$

where we used $\operatorname{vec}(\mathbf{H})^{\top} \operatorname{vec}(\mathbf{H})=n-1$ and

$$
\sum_{i=1}^{n} \operatorname{vec}(\mathbf{H})^{\top}\left(\mathbf{e}_{i} \mathbf{e}_{i}^{\top} \otimes \mathbf{e}_{i} \mathbf{e}_{i}^{\top}\right) \operatorname{vec}(\mathbf{H})=\sum_{i=1}^{n} h_{i i}^{2}=\frac{(n-1)^{2}}{n} .
$$


Next, we find the expression for $\tau_{2}$. For $q \neq r$, we have

$$
\begin{aligned}
(n-1)^{2} \tau_{2}= & \operatorname{cov}\left(\left(\mathbf{Z} \mathbf{e}_{q}\right)^{\top} \mathbf{H}\left(\mathbf{Z} \mathbf{e}_{q}\right),\left(\mathbf{Z} \mathbf{e}_{r}\right)^{\top} \mathbf{H}\left(\mathbf{Z} \mathbf{e}_{r}\right)\right) \\
= & \mathbb{E}\left[\left(\mathbf{Z} \mathbf{e}_{q}\right)^{\top} \mathbf{H}\left(\mathbf{Z} \mathbf{e}_{q}\right)\left(\mathbf{Z} \mathbf{e}_{r}\right)^{\top} \mathbf{H}\left(\mathbf{Z} \mathbf{e}_{r}\right)\right] \\
& -\mathbb{E}\left[\left(\mathbf{Z} \mathbf{e}_{q}\right)^{\top} \mathbf{H}\left(\mathbf{Z} \mathbf{e}_{q}\right)\right] \mathbb{E}\left[\left(\mathbf{Z} \mathbf{e}_{r}\right)^{\top} \mathbf{H}\left(\mathbf{Z} \mathbf{e}_{r}\right)\right] .
\end{aligned}
$$

By using basic algebraic properties of the trace and the vectorization transform and noting that $\mathbb{E}\left[\left(\mathbf{Z} \mathbf{e}_{r}\right)\left(\mathbf{Z} \mathbf{e}_{r}\right)^{\top}\right]=\mathbf{I}$, we arrive at the form

$$
\begin{aligned}
& (n-1)^{2} \tau_{2}= \\
& \operatorname{tr}\left((\mathbf{H} \otimes \mathbf{H}) \mathbb{E}\left[\operatorname{vec}\left(\left(\mathbf{Z} \mathbf{e}_{q}\right)\left(\mathbf{Z} \mathbf{e}_{r}\right)^{\top}\right) \operatorname{vec}\left(\left(\mathbf{Z} \mathbf{e}_{q}\right)\left(\mathbf{Z} \mathbf{e}_{r}\right)^{\top}\right)^{\top}\right]\right) \\
& \quad-\operatorname{tr}(\mathbf{H})^{2} .
\end{aligned}
$$

The expression involving the expectation is equal to $\operatorname{var}\left(\operatorname{vec}\left(\left(\mathbf{Z e}_{q}\right)\left(\mathbf{Z} \mathbf{e}_{r}\right)^{\top}\right)\right)$, which implies

$$
\begin{gathered}
(n-1)^{2} \tau_{2}= \\
\operatorname{tr}\left\{(\mathbf{H} \otimes \mathbf{H})\left(\mathbf{I}+\kappa \sum_{i=1}^{n} \mathbf{e}_{i} \mathbf{e}_{i}^{\top} \otimes \mathbf{e}_{i} \mathbf{e}_{i}^{\top}\right)\right\}-\operatorname{tr}(\mathbf{H})^{2} .
\end{gathered}
$$

By noting that $\operatorname{tr}(\mathbf{H} \otimes \mathbf{H})=\operatorname{tr}(\mathbf{H})^{2}$ and

$$
\sum_{i=1}^{n} \operatorname{tr}\left((\mathbf{H} \otimes \mathbf{H})\left(\mathbf{e}_{i} \mathbf{e}_{i}^{\top} \otimes \mathbf{e}_{i} \mathbf{e}_{i}^{\top}\right)=\sum_{i=1}^{n} h_{i i}^{2},\right.
$$

we find that

$$
\tau_{2}=\frac{1}{(n-1)^{2}} \kappa \frac{(n-1)^{2}}{n}=\frac{\kappa}{n} .
$$

By substituting (16) into (15), and noticing that

$$
\begin{gathered}
\left(\boldsymbol{\Sigma}^{1 / 2} \otimes \boldsymbol{\Sigma}^{1 / 2}\right) \operatorname{vec}(\mathbf{I})=\operatorname{vec}(\boldsymbol{\Sigma}) \text { and } \\
\left(\boldsymbol{\Sigma}^{1 / 2} \otimes \boldsymbol{\Sigma}^{1 / 2}\right)\left(\mathbf{I}+\mathbf{K}_{p}\right)\left(\boldsymbol{\Sigma}^{1 / 2} \otimes \boldsymbol{\Sigma}^{1 / 2}\right)=\left(\mathbf{I}+\mathbf{K}_{p}\right)(\boldsymbol{\Sigma} \otimes \boldsymbol{\Sigma}),
\end{gathered}
$$

completes the proof.

Theorem 2 reveals that the elliptical kurtosis parameter $\kappa$ along with the true covariance matrix $\boldsymbol{\Sigma}$ provide a complete description of the covariances between the elements $s_{i j}$ and $s_{k l}$ of the SCM $\mathbf{S}=\left(s_{i j}\right)$. The mathematics underlying Theorem 2 is so rich that we are able to relate it to at least three fundamental results in the field of statistics given below.

First, consider the one-dimensional case, $p=1$, where we have a univariate sample $x_{1}, \ldots, x_{n}$ from a distribution of a random variable $x \in \mathbb{R}$. Then, the SCM reduces to the unbiased sample variance $s^{2}=(1 /(n-1)) \sum_{i=1}^{n}\left(x_{i}-\bar{x}\right)^{2}$ and equation (14) reduces to $\operatorname{var}\left(s^{2}\right)$. We can now compute $\operatorname{var}\left(s^{2}\right)$ using (14), which states that

$$
\begin{aligned}
\operatorname{var}\left(s^{2}\right) & =\left(\frac{1}{n-1}+\frac{\kappa}{n}\right) 2 \sigma^{4}+\frac{\kappa}{n} \sigma^{4} \\
& =\sigma^{4}\left(\frac{2}{n-1}+\frac{\operatorname{kurt}(x)}{n}\right),
\end{aligned}
$$

where we used that $\boldsymbol{\Sigma} \equiv \sigma^{2}=\mathbb{E}\left[(x-\mathbb{E}[x])^{2}\right], \boldsymbol{\Sigma} \otimes \boldsymbol{\Sigma}=\sigma^{4}$ and $\kappa=\operatorname{kurt}(x) / 3$ due to (13). Hence, we obtained the classic formula for $\operatorname{var}\left(s^{2}\right)$ often encountered in elementary statistics textbooks. Under the Gaussian distribution, $\operatorname{kurt}(x)=0$, in which case Theorem 2 states that $\operatorname{var}\left(s^{2}\right)=2 \sigma^{4} /(n-1)$. This is an expected result since $(n-1) s^{2} / \sigma^{2}=\sum_{i}\left(x_{i}-\bar{x}\right)^{2} / \sigma^{2} \sim$ $\chi_{n-1}^{2}$.

Secondly, we can connect Theorem 2 with the well-known covariance matrix of the Wishart distribution. Let $W_{p}(m, \mathbf{M})$ denote the Wishart distribution of a random symmetric positive definite $p \times p$ matrix where $m>p-1$ denotes the degrees of freedom parameter and $\mathbf{M} \in \mathbb{S}_{++}^{p \times p}$ denotes the scale matrix parameter of the Wishart distribution. Under the MVN assumption, it is well-known that $(n-1) \mathbf{S} \sim W_{p}(n-1, \boldsymbol{\Sigma})$ and consequently $\operatorname{var}(\operatorname{vec}(\mathbf{S}))$ has the famous covariance matrix form

$$
\operatorname{var}(\operatorname{vec}(\mathbf{S}))=\frac{1}{n-1}\left(\mathbf{I}+\mathbf{K}_{p}\right)(\boldsymbol{\Sigma} \otimes \boldsymbol{\Sigma}) .
$$

Suppose now that the elliptical distribution in Theorem 2 is the multivariate normal, thus, $\mathbf{x}_{1}, \ldots, \mathbf{x}_{n} \stackrel{\text { iid }}{\sim} \mathcal{N}_{p}(\boldsymbol{\mu}, \boldsymbol{\Sigma})$. Since in this case $\kappa=0$, we have that (14) reduces to (21).

Lastly, notice that

$\operatorname{var}(\sqrt{n} \operatorname{vec}(\mathbf{S})) \rightarrow(1+\kappa)\left(\mathbf{I}+\mathbf{K}_{p}\right)(\boldsymbol{\Sigma} \otimes \boldsymbol{\Sigma})+\kappa \operatorname{vec}(\boldsymbol{\Sigma}) \operatorname{vec}(\boldsymbol{\Sigma})^{\top}$ as $n \rightarrow \infty$. The right hand side of the previous equation equals the well-known asymptotic covariance matrix of the limiting normal distribution of $\sqrt{n}(\operatorname{vec}(\mathbf{S})-\operatorname{vec}(\boldsymbol{\Sigma}))$ when sampling from an elliptical distribution $\mathcal{E}_{p}(\boldsymbol{\mu}, \boldsymbol{\Sigma}, g)$ with finite fourth order moments. This is a famous result in multivariate statistics [14].

In the next Lemma, we derive the MSE and normalized MSE of the SCM.

Lemma 1. Let $\mathbf{x}_{1}, \ldots, \mathbf{x}_{n}$ denote an i.i.d. random sample from a p-variate elliptical distribution with finite fourth order moments, mean $\mu$, and covariance matrix $\Sigma$. Then, the MSE and the NMSE of $\mathbf{S}$ are

$$
\begin{aligned}
\operatorname{MSE}(\mathbf{S}) & =\left(\frac{1}{n-1}+\frac{\kappa}{n}\right) \operatorname{tr}(\boldsymbol{\Sigma})^{2}+\left(\frac{1}{n-1}+\frac{2 \kappa}{n}\right) \operatorname{tr}\left(\boldsymbol{\Sigma}^{2}\right) \\
\operatorname{NMSE}(\mathbf{S}) & =\left(1+\frac{p}{\gamma}\right)\left(\frac{1}{n-1}+\frac{\kappa}{n}\right)+\frac{\kappa}{n}
\end{aligned}
$$

where $\gamma$ and $\kappa$ are defined in (3) and (13), respectively.

Proof. Since $\mathbf{S}$ is unbiased, i.e., $\mathbb{E}[\mathbf{S}]=\boldsymbol{\Sigma}$, it holds that

$$
\begin{aligned}
\operatorname{MSE}(\mathbf{S}) & =\mathbb{E}\left[\|\mathbf{S}-\mathbf{\Sigma}\|_{\mathrm{F}}^{2}\right] \\
& =\mathbb{E}\left[\operatorname{vec}(\mathbf{S}-\mathbf{\Sigma})^{\top} \operatorname{vec}(\mathbf{S}-\mathbf{\Sigma})\right] \\
& =\operatorname{tr}\left(\mathbb{E}\left[\operatorname{vec}(\mathbf{S}-\mathbb{E}[\mathbf{S}]) \operatorname{vec}(\mathbf{S}-\mathbb{E}[\mathbf{S}])^{\top}\right]\right) \\
& =\operatorname{tr}(\operatorname{var}(\operatorname{vec}(\mathbf{S}))),
\end{aligned}
$$

Then we substitute the expression stated in (14) for $\operatorname{var}(\operatorname{vec}(\mathbf{S}))$ into equation (22) and use the following identities

$$
\begin{aligned}
\operatorname{tr}(\boldsymbol{\Sigma} \otimes \boldsymbol{\Sigma}) & =\operatorname{tr}(\boldsymbol{\Sigma})^{2}, \\
\operatorname{tr}\left(\operatorname{vec}(\boldsymbol{\Sigma}) \operatorname{vec}(\boldsymbol{\Sigma})^{\top}\right) & =\operatorname{tr}\left(\boldsymbol{\Sigma}^{2}\right), \text { and } \\
\operatorname{tr}\left(\mathbf{K}_{p}(\boldsymbol{\Sigma} \otimes \boldsymbol{\Sigma})\right) & =\operatorname{tr}\left(\boldsymbol{\Sigma}^{2}\right)
\end{aligned}
$$

where the last identity follows from

$$
\begin{aligned}
\operatorname{tr}\left(\mathbf{K}_{p}(\boldsymbol{\Sigma} \otimes \boldsymbol{\Sigma})\right) & =\sum_{i, j} \operatorname{tr}\left(\left(\mathbf{e}_{i} \mathbf{e}_{j}^{\top} \otimes \mathbf{e}_{j} \mathbf{e}_{i}^{\top}\right)(\boldsymbol{\Sigma} \otimes \boldsymbol{\Sigma})\right) \\
& =\sum_{i, j} \operatorname{tr}\left(\mathbf{e}_{j}^{\top} \boldsymbol{\Sigma} \mathbf{e}_{i} \cdot \mathbf{e}_{i}^{\top} \boldsymbol{\Sigma} \mathbf{e}_{j}\right) \\
& =\operatorname{tr}\left(\boldsymbol{\Sigma}^{2}\right),
\end{aligned}
$$


to obtain the stated expression of the $\operatorname{MSE}(\mathbf{S})$. The expression for the $\operatorname{NMSE}(\mathbf{S})$ is obtained by dividing the $\operatorname{MSE}(\mathbf{S})$ by $\|\boldsymbol{\Sigma}\|_{\mathrm{F}}^{2}=\operatorname{tr}\left(\boldsymbol{\Sigma}^{2}\right)$.

The next theorem states that the oracle parameters derived in Theorem 1 can be written in a much simpler form when sampling from an elliptically symmetric distribution.

Theorem 3. Let $\mathbf{x}_{1}, \ldots, \mathbf{x}_{n}$ denote an i.i.d. random sample from an elliptical distribution with finite fourth order moments, mean $\boldsymbol{\mu}$, and covariance matrix $\boldsymbol{\Sigma}$. Then the oracle parameters $\left(\alpha_{o}, \beta_{o}\right)$ that minimize the MSE are

$$
\beta_{o}^{\mathrm{Ell}}=\frac{(\gamma-1)}{(\gamma-1)+\kappa(2 \gamma+p) / n+(\gamma+p) /(n-1)},
$$

and $\alpha_{o}^{\mathrm{Ell}}=\left(1-\beta_{o}^{\mathrm{Ell}}\right) \eta$, where the parameters $\eta$, $\gamma$ and $\kappa$ are defined in (3) and (13), respectively.

Proof. Follows from (5) and Lemma 1.

It is not surprising that $\beta_{o}$, and hence also $\alpha_{o}$, depend on the density generator $g$ of the elliptical distribution only via the elliptical kurtosis parameter $\kappa$. Specifying the elliptical distribution also specifies the value of $\kappa$. For example, when sampling from the Gaussian distribution, the elliptical kurtosis parameter is $\kappa=0$ and $\beta_{o}^{\text {Ell }}$ in Theorem 3 reduces to

$$
\beta_{o}^{\text {Gau }}=\frac{(\gamma-1)}{(\gamma-1)+(\gamma+p) /(n-1)} .
$$

Consequently, an estimator of $\beta_{o}^{\mathrm{Gau}}$ is obtained by substituting an estimator $\hat{\gamma}$ in place of $\gamma$ in (23). Recall that an estimator of $\alpha_{o}^{\mathrm{Gau}}$ is then obtained as $\hat{\alpha}_{o}^{\mathrm{Gau}}=\left(1-\hat{\beta}_{o}^{\mathrm{Gau}}\right) \operatorname{tr}(\mathbf{S}) / p$.

Since in this paper we do not assume any particular elliptical distribution, we need to find an estimator $\hat{\kappa}$ of the elliptical kurtosis parameter $\kappa$ as well. Naturally, if the assumption on multivariate normality of the data is valid, then (23) should be used for estimating the optimal oracle value.

When the mean vector of the population is known, the unbiased SCM is $\mathbf{S}=\frac{1}{n} \sum_{i=1}^{n} \mathbf{x}_{i} \mathbf{x}_{i}^{\top}$ (as one can assume without loss of generality that $\boldsymbol{\mu}=\mathbf{0}$ ). In this case the optimal shrinkage parameter $\beta_{o}$ of the RSCM stated in Theorem 3 remains unchanged apart from the last term in the denominator of $\beta_{o}$, that is, $(\gamma+p) /(n-1)$ is replaced by $(\gamma+p) / n$. This centered case was addressed in [20].

\section{ESTIMATION OF THE ORACLE PARAMETERS}

In this section, we develop estimators $\hat{\gamma}$ and $\hat{\kappa}$ of the unknown parameters $\kappa$ and $\gamma$ that determine the shrinkage parameter $\beta_{o}$ (cf. Theorem 3). These are used to obtain a plug-in estimators of the shrinkage parameters as

$$
\begin{aligned}
& \hat{\beta}_{o}^{\text {Ell }}=\frac{(\hat{\gamma}-1)}{(\hat{\gamma}-1)+\hat{\kappa}(2 \hat{\gamma}+p) / n+(\hat{\gamma}+p) /(n-1)}, \\
& \hat{\alpha}_{o}^{\text {Ell }}=\left(1-\hat{\beta}_{o}^{\text {Ell }}\right) \hat{\eta} .
\end{aligned}
$$

Next, we will address how to estimate the needed statistical parameters. First, we will address the estimation of $\kappa$. Regarding $\gamma$, we found two different well performing estimators, and hence, we will address its estimation last.
A natural estimate of $\kappa$ is the conventional sample average

$$
\hat{\kappa}=\max \left(-\frac{2}{p+2}, \frac{1}{3 p} \sum_{j=1}^{p} \hat{K}_{j}\right),
$$

where $\hat{K}_{j}$ is an estimate of the kurtosis of the $j$ th variable and defined as

$$
\hat{K}_{j}=\frac{n-1}{(n-2)(n-3)}\left((n+1) \hat{k}_{j}+6\right) .
$$

Here $\hat{k}_{j}=m_{j}^{(4)} /\left(m_{j}^{(2)}\right)^{2}-3$ denotes the conventional sample estimate of the kurtosis of the $j$ th variable, where $m_{j}^{(q)}=$ $\frac{1}{n} \sum_{i=1}^{n}\left(x_{i j}-\bar{x}_{j}\right)^{q}$ denotes the $q$ th order sample moment. The estimate $\hat{K}_{j}$ is a commonly used estimate of the kurtosis which is based on the relationship between the kurtosis and the cumulants of the distribution [21]. It corrects for the bias of the conventional sample kurtosis $\hat{k}_{j}$. To ensure that the final estimate $\hat{\kappa}$ does not go below the theoretical lower bound of $-2 /(p+2)$ [22], a maximum constraint is used in (24). The constructed estimate of $\kappa$ is consistent both in the conventional and the RMT regime.

Note that, if the estimates $(\hat{\gamma}, \hat{\kappa})$ are restricted to be within their theoretical ranges, i.e., $1 \leq \hat{\gamma} \leq p$ and $\hat{\kappa} \geq-2 /(p+2)$, then it is straightforward to verify that the plug-in estimator satisfies $\hat{\beta}_{o}^{\text {Ell }} \in[0,1)$.

In the following subsections, we consider two options for estimating the sphericity $\gamma$ under the RMT regime. We denote the estimators by $\hat{\gamma}^{\text {Ell1 }}$ and $\hat{\gamma}^{\text {Ell2 }}$. Both estimators have their own benefits and disadvantages. The first estimator, $\hat{\gamma}^{\text {Ell1 }}$, enjoys statistical robustness with respect to heavier-tailed distributions. The second estimator, $\hat{\gamma}^{\text {Ell2 }}$, is computationally more efficient and can easily be used and tuned for very highdimensional set-ups such as microarray studies where $p$ is often tens of thousands but $n$ is of few tens [23]. It is also highly efficient under Gaussianity, or for mild departures from Gaussianity. Its obvious disadvantage is that it is not very efficient for heavier-tailed elliptical distributions.

\section{A. Ell1-RSCM estimator}

The first estimator of the sphericity $\gamma$, uses the sample spatial sign covariance matrix, defined as

$$
\mathbf{S}_{\mathrm{sgn}}=\frac{1}{n} \sum_{i=1}^{n} \frac{\left(\mathbf{x}_{i}-\hat{\boldsymbol{\mu}}\right)\left(\mathbf{x}_{i}-\hat{\boldsymbol{\mu}}\right)^{\top}}{\left\|\mathbf{x}_{i}-\hat{\boldsymbol{\mu}}\right\|^{2}},
$$

where $\hat{\boldsymbol{\mu}}=\arg \min _{\boldsymbol{\mu}} \sum_{i=1}^{n}\left\|\mathbf{x}_{i}-\boldsymbol{\mu}\right\|$ is the sample spatial median [24]. The sample sign covariance matrix is well-known to be highly robust although it is not a consistent estimator of the covariance matrix [25], [26]. Namely, it does provide consistent estimators of the eigenvectors of the covariance matrix but not of the eigenvalues.

Consider an estimator of the form,

$$
\begin{aligned}
\hat{\gamma}^{\mathrm{Ell1*}} & =\frac{n}{n-1}\left(p \operatorname{tr}\left(\mathbf{S}_{\mathrm{sgn}}^{2}\right)-\frac{p}{n}\right) \\
& =\frac{p}{n(n-1)} \sum_{i \neq j}\left(\mathbf{v}_{i}^{\top} \mathbf{v}_{j}\right)^{2} \\
& =p \operatorname{ave}_{i \neq j}\left\{\cos ^{2}\left(\varangle\left(\mathbf{x}_{i}, \mathbf{x}_{j}\right)\right)\right\}
\end{aligned}
$$


where ave $e_{i, j}$ denotes arithmetic average over indices, $i, j \in$ $\{1, \ldots, n\}, i \neq j$, and $\mathbf{v}_{i}=\left(\mathbf{x}_{i}-\hat{\boldsymbol{\mu}}\right) /\left\|\mathbf{x}_{i}-\hat{\boldsymbol{\mu}}\right\|$.

In [5, Lemma 4.1] it was shown that $\hat{\gamma}^{\text {Ell1* }}$ is a consistent estimator of $\gamma$ when sampling from a centered elliptical distribution $\mathcal{E}_{p}(\mathbf{0}, \boldsymbol{\Sigma}, g)$ under the RMT regime (R1) and when the eigenvalues of $\Sigma$ converge to a fixed spectrum such that

$$
\begin{array}{r}
\operatorname{tr}\left(\boldsymbol{\Sigma}^{i}\right) / p \text { has a finite and positive limit } \\
\text { for } i=1,2,3,4 \text { as } p \rightarrow \infty .
\end{array}
$$

In their paper, it was assumed that the location (symmetry center) is known to be zero, which is why they do not have the centering of the samples by the sample spatial median in (25). We also remark that our estimator $\hat{\gamma}^{\text {Ell1* }}$ differs from [5, Lemma 4.1] in that we scale their estimator by $\frac{n}{n-1}$. This scaling is used for correcting bias for small samples and needed to ensure that $\mathbb{E}\left[\hat{\gamma}^{\mathrm{E} I 11^{*}}\right] \in[1, p]$. In order to guarantee that the estimate remains inside the valid interval $[1, p]$, as a final estimator, we use

$$
\hat{\gamma}^{\mathrm{E} I 11}=\min \left(p, \max \left(1, \hat{\gamma}^{\mathrm{Ell1}}\right)\right) .
$$

We can now define the Ell1-RSCM estimator as the RSCM based on the estimators of the optimal shrinkage parameters using the plugin estimates $\hat{\eta}$ of (11), $\hat{\kappa}$ of (24) and $\hat{\gamma}^{\mathrm{Ell1}}$ of (26).

\section{B. Ell2-RSCM estimator}

In order to develop the second estimator $\hat{\gamma}^{\text {Ell2 }}$ of $\gamma$, we need to find the values of $\mathbb{E}\left[\operatorname{tr}\left(\mathbf{S}^{2}\right)\right]$ and $\mathbb{E}\left[\operatorname{tr}(\mathbf{S})^{2}\right]$, which are given in the following Lemma 2.

Lemma 2. Let $\mathbf{x}_{1}, \ldots, \mathbf{x}_{n}$ denote an i.i.d. random sample from an elliptical distribution with finite fourth order moments, mean vector $\boldsymbol{\mu}$, and covariance matrix $\boldsymbol{\Sigma}$. Then,

$$
\begin{aligned}
& \mathbb{E}\left[\operatorname{tr}\left(\mathbf{S}^{2}\right)\right]= \\
& \quad\left(\frac{1}{n-1}+\frac{\kappa}{n}\right) \operatorname{tr}(\boldsymbol{\Sigma})^{2}+\left(1+\frac{1}{n-1}+\frac{2 \kappa}{n}\right) \operatorname{tr}\left(\boldsymbol{\Sigma}^{2}\right)
\end{aligned}
$$

and

$$
\mathbb{E}\left[\operatorname{tr}(\mathbf{S})^{2}\right]=\left(1+\frac{\kappa}{n}\right) \operatorname{tr}(\boldsymbol{\Sigma})^{2}+2\left(\frac{1}{n-1}+\frac{\kappa}{n}\right) \operatorname{tr}\left(\boldsymbol{\Sigma}^{2}\right)
$$

Proof. The first statement for $\mathbb{E}\left[\operatorname{tr}\left(\mathbf{S}^{2}\right)\right]$ follows from Lemma 1 by noting that $\mathbb{E}\left[\operatorname{tr}\left(\mathbf{S}^{2}\right)\right]=\operatorname{MSE}(\mathbf{S})+\operatorname{tr}\left(\boldsymbol{\Sigma}^{2}\right)$, which was shown in (9).

Regarding the second statement, we first write

$$
\begin{aligned}
\mathbb{E}\left[\operatorname{tr}(\mathbf{S})^{2}\right] & =\mathbb{E}\left[\sum_{i} s_{i i} \sum_{j} s_{j j}\right]=\sum_{i, j} \mathbb{E}\left[s_{i i} s_{j j}\right] \\
& =\sum_{i, j}\left(\operatorname{cov}\left(s_{i i}, s_{j j}\right)+\mathbb{E}\left[s_{i i}\right] \mathbb{E}\left[s_{j j}\right]\right) .
\end{aligned}
$$

Here, the covariance of $s_{i i}$ and $s_{j j}$ is the $i j$ th element of the $i j$ th block of $\operatorname{var}(\operatorname{vec}(\mathbf{S}))$ in (14) since

$$
\begin{aligned}
\operatorname{cov}\left(s_{i i}, s_{j j}\right) & =\operatorname{cov}\left(\mathbf{e}_{i}^{\top} \mathbf{S} \mathbf{e}_{i}, \mathbf{e}_{j}^{\top} \mathbf{S} \mathbf{e}_{j}\right) \\
& =\operatorname{cov}\left(\left(\mathbf{e}_{i} \otimes \mathbf{e}_{i}\right)^{\top} \operatorname{vec}(\mathbf{S}),\left(\mathbf{e}_{j} \otimes \mathbf{e}_{j}\right)^{\top} \operatorname{vec}(\mathbf{S})\right) \\
& =\left(\mathbf{e}_{i}^{\top} \otimes \mathbf{e}_{i}^{\top}\right) \operatorname{var}(\operatorname{vec}(\mathbf{S}))\left(\mathbf{e}_{j} \otimes \mathbf{e}_{j}\right) .
\end{aligned}
$$

Using the following identities:

$$
\begin{aligned}
\left(\mathbf{e}_{i}^{\top} \otimes \mathbf{e}_{i}^{\top}\right)(\boldsymbol{\Sigma} \otimes \boldsymbol{\Sigma})\left(\mathbf{e}_{j} \otimes \mathbf{e}_{j}\right) & =\mathbf{e}_{i}^{\top} \boldsymbol{\Sigma} \mathbf{e}_{j} \cdot \mathbf{e}_{i}^{\top} \boldsymbol{\Sigma} \mathbf{e}_{j}, \\
\left(\mathbf{e}_{i}^{\top} \otimes \mathbf{e}_{i}^{\top}\right) \mathbf{K}_{p}(\boldsymbol{\Sigma} \otimes \boldsymbol{\Sigma})\left(\mathbf{e}_{j} \otimes \mathbf{e}_{j}\right) & =\mathbf{e}_{i}^{\top} \boldsymbol{\Sigma} \mathbf{e}_{j} \cdot \mathbf{e}_{i}^{\top} \boldsymbol{\Sigma} \mathbf{e}_{j}, \\
\left(\mathbf{e}_{i}^{\top} \otimes \mathbf{e}_{i}^{\top}\right) \operatorname{vec}(\boldsymbol{\Sigma}) \operatorname{vec}(\boldsymbol{\Sigma})^{\top}\left(\mathbf{e}_{j} \otimes \mathbf{e}_{j}\right) & =\mathbf{e}_{i}^{\top} \boldsymbol{\Sigma} \mathbf{e}_{i} \cdot \mathbf{e}_{j}^{\top} \boldsymbol{\Sigma} \mathbf{e}_{j},
\end{aligned}
$$

and the fact that $\mathbf{S}$ is unbiased, i.e., $\mathbb{E}\left[s_{i i}\right]=\mathbf{e}_{i}^{\top} \boldsymbol{\Sigma} \mathbf{e}_{i}$, we can write using (14) that

$$
\begin{aligned}
\mathbb{E}\left[s_{i i} s_{j j}\right] & =\operatorname{cov}\left(s_{i i}, s_{j j}\right)+\mathbb{E}\left[s_{i i}\right] \mathbb{E}\left[s_{j j}\right] \\
& =2 \tau_{1}\left(\mathbf{e}_{i}^{\top} \boldsymbol{\Sigma} \mathbf{e}_{j} \cdot \mathbf{e}_{i}^{\top} \boldsymbol{\Sigma} \mathbf{e}_{j}\right)+\left(1+\tau_{2}\right)\left(\mathbf{e}_{i}^{\top} \boldsymbol{\Sigma} \mathbf{e}_{i} \cdot \mathbf{e}_{j}^{\top} \boldsymbol{\Sigma} \mathbf{e}_{j}\right),
\end{aligned}
$$

where $\tau_{1}$ and $\tau_{2}$ are given in (18) and (19), respectively. By summing all $i$ and $j$, we have

$$
\mathbb{E}\left[\operatorname{tr}(\mathbf{S})^{2}\right]=\sum_{i, j} \mathbb{E}\left[s_{i i} s_{j j}\right]=2 \tau_{1} \operatorname{tr}\left(\boldsymbol{\Sigma}^{2}\right)+\left(1+\tau_{2}\right) \operatorname{tr}(\boldsymbol{\Sigma})^{2},
$$

which completes the proof.

Next, we construct an estimator for $\vartheta=\operatorname{tr}\left(\boldsymbol{\Sigma}^{2}\right) / p$. The natural plug-in estimate, $\operatorname{tr}\left(\mathbf{S}^{2}\right) / p$, is not a consistent estimator in the RMT regime (R1) and (R2). This follows at once from Lemma 2 as it shows that $\operatorname{tr}\left(\mathbf{S}^{2}\right) / p$ is not asymptotically unbiased since

$$
\lim _{\substack{n, p \rightarrow \infty \\ p / n \rightarrow c_{o}}} \frac{\mathbb{E}\left[\operatorname{tr}\left(\mathbf{S}^{2}\right)\right]}{p}=c_{0}(1+\kappa) \eta_{o}^{2}+\vartheta_{0},
$$

where $\eta_{o}>0$ and $\vartheta_{o}>0$ denote finite limit values of $\operatorname{tr}(\boldsymbol{\Sigma}) / p$ and $\operatorname{tr}\left(\boldsymbol{\Sigma}^{2}\right) / p$, respectively, as $p \rightarrow \infty$.

In the next Theorem 4, a proper estimator $\hat{\vartheta}$ of $\vartheta$ under the RMT regime is developed. Theorem 4 extends [18, Lemma 2.1] to the elliptical case.

Theorem 4. Let $\mathrm{x}_{1}, \ldots, \mathrm{x}_{n}$ denote an i.i.d. random sample from a p-variate elliptical distribution with finite fourth order moments, mean vector $\boldsymbol{\mu}$, and covariance matrix $\boldsymbol{\Sigma}$. Then, an unbiased estimate of $\vartheta=\operatorname{tr}\left(\boldsymbol{\Sigma}^{2}\right) / p$ for any finite $n$ and any $p$ is

$$
\hat{\vartheta}=b_{n}\left(\frac{\operatorname{tr}\left(\mathbf{S}^{2}\right)}{p}-a_{n} \frac{p}{n}\left[\frac{\operatorname{tr}(\mathbf{S})}{p}\right]^{2}\right)
$$

where

$$
\begin{aligned}
& a_{n}=\left(\frac{n}{n+\kappa}\right)\left(\frac{n}{n-1}+\kappa\right) \\
& b_{n}=\frac{(\kappa+n)(n-1)^{2}}{(n-2)(3 \kappa(n-1)+n(n+1))} .
\end{aligned}
$$

Furtheremore, under the RMT regime $(R 1)$ and $(R 2)$, the estimator is asymptotically unbiased, i.e., $\mathbb{E}[\hat{\vartheta}] \rightarrow \vartheta_{o}$, where $\vartheta_{o}>0$ denotes the finite limit of $\vartheta$ as $p \rightarrow \infty$.

Proof. Using Lemma 2 write

$$
\begin{aligned}
& b_{n}^{-1} p \mathbb{E}[\hat{\vartheta}]= \\
& \left(\tau_{1}-\frac{a_{n}}{n}\left(1+\tau_{2}\right)\right) \operatorname{tr}(\boldsymbol{\Sigma})^{2}+\left(1+\tau_{1}+\tau_{2}-2 \tau_{1} \frac{a_{n}}{n}\right) \operatorname{tr}\left(\boldsymbol{\Sigma}^{2}\right),
\end{aligned}
$$

where $\tau_{1}$ and $\tau_{2}$ are defined in (18) and (19). By choosing $a_{n}=n \tau_{1} /\left(1+\tau_{2}\right)$, we see that $b_{n}=\left(1+\tau_{1}+\tau_{2}-2 \tau_{1} a_{n} / n\right)^{-1}$. The terms $a_{n}$ and $b_{n}$ can equivalently be expressed in the form given in the theorem by using the equations for $\tau_{1}$ and $\tau_{2}$. The 
last statement is a consequence of the fact that $\vartheta$ converges to a finite limit value as $p \rightarrow \infty$ and that $\mathbb{E}[\hat{\vartheta}]=\vartheta$.

Note that $a_{n}$ and $b_{n}$ depend on the elliptical distribution via the elliptical kurtosis parameter $\kappa$. Using the estimate of the kurtosis by defining $\hat{a}_{n}=a_{n}(\hat{\kappa})$ and $\hat{b}_{n}=b_{n}(\hat{\kappa})$ one obtains an estimate of $\vartheta$ which does not require knowing the underlying elliptical distribution. Thus based on Theorem 4, we propose an estimator of the form

$$
\hat{\gamma}^{\mathrm{EII2*}}=\hat{b}_{n}\left(\frac{p \operatorname{tr}\left(\mathbf{S}^{2}\right)}{\operatorname{tr}(\mathbf{S})^{2}}-\hat{a}_{n} c\right) .
$$

Note that, if $n$ is reasonably large (e.g., $n>100$ ), then $\hat{a}_{n} \approx$ $1+\hat{\kappa}$ and $b_{n} \approx 1$ and then one may use

$$
\hat{\gamma}^{\mathrm{Ell} 2 *}=\left(\frac{p \operatorname{tr}\left(\mathbf{S}^{2}\right)}{\operatorname{tr}(\mathbf{S})^{2}}-(1+\hat{\kappa}) c\right) .
$$

In order to guarantee that the estimator remains in the valid interval, $1 \leq \gamma \leq p$, we use

$$
\hat{\gamma}^{\mathrm{Ell} 2}=\min \left(p, \max \left(1, \hat{\gamma}^{\mathrm{Ell2*}}\right)\right) .
$$

as our final estimator. We can now define the Ell2-RSCM estimator as the RSCM, which uses $\hat{\eta}$ of (11), $\hat{\kappa}$ of (24), and $\hat{\gamma}^{\mathrm{E} l 2}$ of (28) as plug-in estimates for the optimal shrinkage parameters.

Finally, we wish to note that albeit $\hat{\gamma}^{\text {Ell2 }}$ does not require knowledge of the underlying elliptically symmetric distribution of the data, it is not a robust estimator. This is due to the fact that $\operatorname{tr}\left(\mathbf{S}^{2}\right)$ contains 4 th order moments of the data, and 8th order moments of the elliptically symmetric distribution needs to exists in order for $\operatorname{tr}\left(\mathbf{S}^{2}\right)$ to be asymptotically normal. Consequently, the Ell2-RSCM estimator is not well suited for heavier-tailed distributions.

\section{Ell3-RSCM estimator}

Ell3-RSCM is a hybrid of the Ell1-RSCM and Ell2-RSCM estimators. The Ell3-RSCM will use the estimator which has a smaller estimated sphericity $\hat{\gamma}$. Thus, it will always favor more shrinkage over less shrinkage. This rule can be summarized as: if $\hat{\gamma}^{\mathrm{Ell1}}<\hat{\gamma}^{\mathrm{Ell} 2}$, then choose Ell1-RSCM, otherwise choose Ell2-RSCM.

\section{Simulation STUdY}

We conduct a small simulation study to investigate the performance of the RSCM estimators in terms of their finite sample NMSE. Each simulation is repeated 10000 times and the NMSE is averaged over the Monte-Carlo runs for each RSCM estimator. The theoretical oracle MSE value derived in (7) is normalized by $\|\boldsymbol{\Sigma}\|_{\mathrm{F}}^{2}$ and used as a benchmark for the empirical NMSE values, which is shown in the figures as a solid black line. The mean vector $\boldsymbol{\mu}$ is fixed for each MC trial and generated randomly as $\left\{\mu_{i}\right\}_{i=1}^{p} \stackrel{i i d}{\sim} \mathcal{N}(0,4)$.

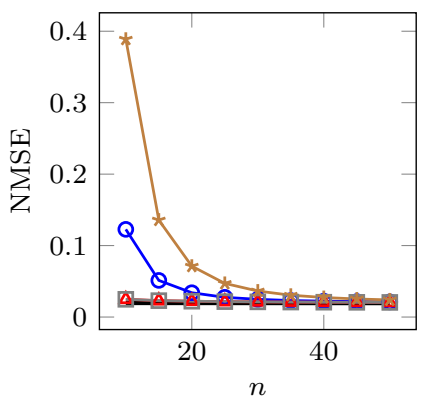

(a) $\varrho=0.1$

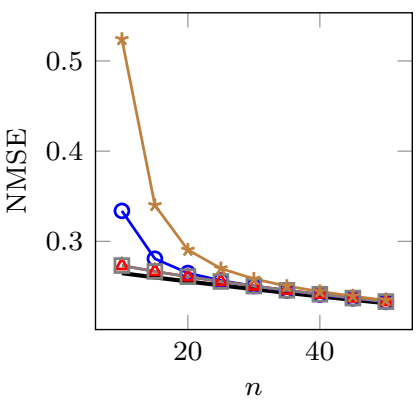

(b) $\varrho=0.4$

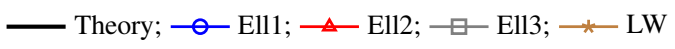

Fig. 1. AR(1) process: comparison of covariance estimators when $p=100$, $\varrho \in\{0.1,0.4\}$, and the samples are from a Gaussian distribution.

\section{A. AR(1) covariance matrix}

In the first experiment, an autoregressive covariance structure is used. We let $\boldsymbol{\Sigma}$ be the covariance matrix of a Gaussian $\operatorname{AR}(1)$ process,

$$
(\boldsymbol{\Sigma})_{i j}=\varrho^{|i-j|}, \quad \text { where } \varrho \in(0,1) .
$$

Note that, $\boldsymbol{\Sigma}$ verifies $\eta=\operatorname{tr}(\boldsymbol{\Sigma}) / p=1$. Also, when $\varrho \downarrow 0$, then $\boldsymbol{\Sigma}$ is close to an identity matrix, and when $\varrho \uparrow 1, \boldsymbol{\Sigma}$ tends to a singular matrix of rank 1 . The dimension is fixed at $p=100$ and $n$ varies from 10 to 50 in steps of 5 samples. Figure 1 depicts the NMSE performance as a function of sample length $n$ when the samples were drawn from a Gaussian distribution.

It can be noted that when the sample sizes were small, both the Ell1-RSCM estimator and the El12-RSCM estimator outperformed the LW-RSCM estimator with a significant margin. We also notice that the performance of the Ell2-RSCM and Ell3-RSCM estimators were almost overlapping with the theoretical optimal value for all values of $n$ and for both values of $\varrho$.

Next, we consider heavier-tailed distributions than the Gaussian. Namely, the $t_{\nu}$-distribution with $\nu=12$ and $\nu=7$ degrees of freedom; the kurtosis of the marginal variable being $\operatorname{kurt}\left(x_{i}\right)=0.75$ and $\operatorname{kurt}\left(x_{i}\right)=2$, respectively. The results are given in Figure 2.

First, we notice that Ell1-RSCM and Ell3-RSCM outperformed Ell2-RSCM and LW-RSCM for all values of $c=p / n$, $\nu$ and $\rho$. In the case of $\nu=7$, the performance of the Ell2RSCM estimator declined due to its non-robustness, and it is performing the worst among the shrinkage estimators. In the case of $\nu=12$, the LW-RSCM estimator and the Ell2RSCM estimator had similar performances for larger values of $n$, but Ell2-RSCM performed better at small values of $n$. Since Ell1-RSCM and Ell2-RSCM differ only in the way they estimate the sphericity $\gamma$, the performance loss of Ell2RSCM over Ell1-RSCM can be attributed to a larger variability and the non-robustness of the estimator $\hat{\gamma}^{\mathrm{Ell} 2}$ as compared to $\hat{\gamma}^{\text {Ell1 }}$. Also note that when the samples were drawn from the $t_{7}$-distribution, the performance loss of LW-RSCM to Ell1RSCM and Ell3-RSCM increased. Indeed, this difference in performance can be attributed to better robustness properties 


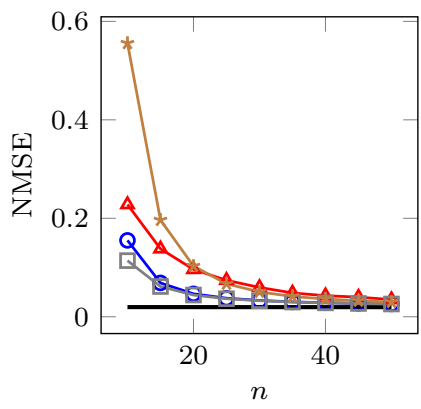

(a) $\varrho=0.1$ and $\nu=12$

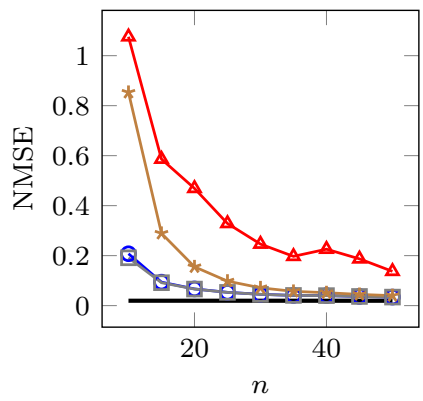

(c) $\varrho=0.1$ and $\nu=7$

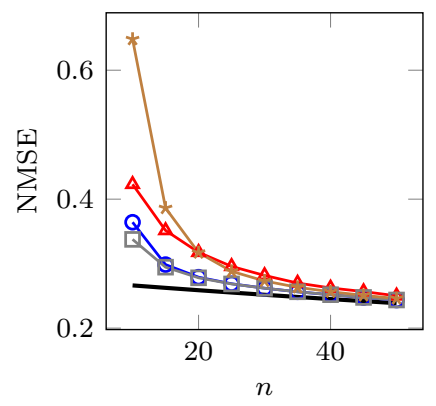

(b) $\varrho=0.4$ and $\nu=12$

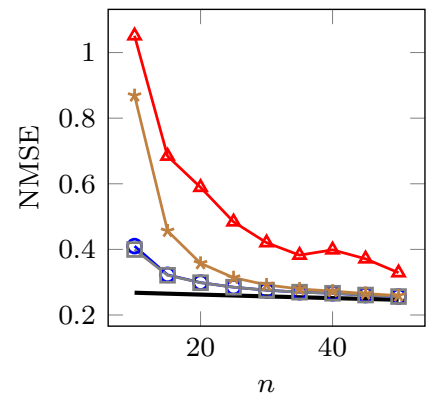

(d) $\varrho=0.4$ and $\nu=7$

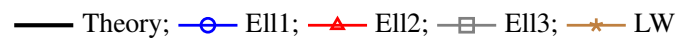

Fig. 2. AR(1) process: comparison of covariance estimators when $p=100$, $\varrho \in\{0.1,0.4\}$, and the samples are drawn from a $t_{\nu}$-distribution.

of the Ell1-RSCM estimator over the LW-RSCM estimator when sampling from a heavier-tailed elliptical distribution.

Finally, for the case $\rho=0.4$ and $n=20$, the Figure 3 depicts the theoretical NMSE of $\mathbf{S}_{(1-\beta) \eta, \beta}$ as a function of $\beta$. Notice that the minimum NMSE is obtained at $\beta_{o}^{\text {Ell }}$ which is shown by black vertical line. The average estimates of the optimal value $\beta_{o}^{\text {Ell }}$ given by the different estimators are also indicated by vertical lines.

As can be seen, for Gaussian data, the Ell2-RSCM estimator of $\beta_{o}$ was very close to the theoretical minimum, and significantly better than the Ell1-RSCM estimator. The LWRSCM estimator was far apart from the minimum compared to Ell1-RSCM and Ell2-RSCM. In the case of the $t_{\nu}$-distribution with $\nu=12$ degrees of freedom, the Ell1-RSCM estimator was performing better than Ell2-RSCM due to its robustness, and both were significantly closer to the minimum than the LW-RSCM estimator.

\section{B. Largely varying spectrum}

The next study follows the set-up in [5], where $\Sigma$ has one (or a few) large eigenvalues. In the first set-up, $\Sigma$ is a diagonal matrix of size $50 \times 50$, where $m$ eigenvalues are equal to 1 and the remaining $50-m$ eigenvalues are equal to 0.01 . For the case $n=10$, Figure 4 depicts the NMSE as a function of $m$ averaged over 10000 Monte Carlo runs when sampling from a Gaussian distribution and a $t_{\nu}$-distribution with $\nu=10$ degrees of freedom.

In the Gaussian case, the Ell2-RSCM estimator had excellent performance as its NMSE curve is essentially overlapping

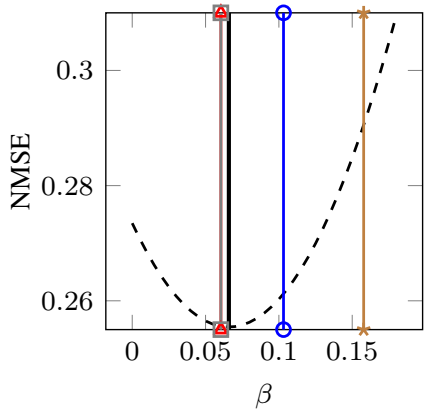

(a) Gaussian samples

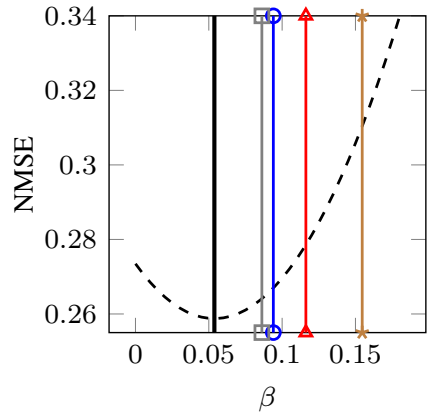

(b) $t_{12}$-distributed samples

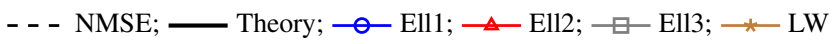

Fig. 3. The theoretical NMSE of the shrinkage estimator $\mathbf{S}_{(1-\beta) \eta, \beta}$ as a function of $\beta$ when the covariance matrix has an $\operatorname{AR}(1)$ structure with $\rho=$ $0.4, p=100$ and $n=20$. The minimum NMSE is obtained at $\beta_{o}^{\text {Ell }}$ which is indicated by a solid vertical line. The average estimated value of the shrinkage parameter $\beta$ obtained by LW-, Ell1-, Ell2, and Ell3-estimators are shown.

with the theoretical NMSE curve. This is attested also in the right-hand side plot which depicts the graph of the average estimate $\hat{\beta}_{o}$ and the theoretical optimal value $\beta_{o}$ as a function of $m$. As can be seen, the Ell2-RSCM estimator was essentially performing at the oracle level, whereas the shrinkage parameter corresponding to the LW-RSCM estimator was somewhat far from the theoretical optimal. The NMSE curves show that the Ell2-RSCM estimator performed better than the Ell1-RSCM estimator for Gaussian samples, however, with a rather small margin. In the case of $t_{10}$-distribution, as expected, Ell1-RSCM performed better than Ell2-RSCM due to its robustness in estimating the sphericity. The hybrid estimator Ell3-RSCM was able to perform slightly better than the other estimators in both cases.

The next simulation set-up considers a very challenging scenario in which the spectrum of $\boldsymbol{\Sigma}$ consists of several different eigenvalues. We consider the case that $p=100$ and the covariance matrix $\Sigma$ has 30 eigenvalues equal to 100 , 40 eigenvalues equal to 1 , and 30 eigenvalues of 0.01 . The samples were drawn from a Gaussian distribution and a $t_{\nu^{-}}$ distribution with $\nu=10$ degrees of freedom. The NMSE curves are plotted as a function of the sample length $n$ in Figure 5.

It can be seen that under Gaussian sampling, the Ell2RSCM and the Ell3-RSCM estimators achieved near optimal performance for all $n$ considered. Indeed, this behavior was already seen in the other simulation studies. The more robust Ell1-RSCM estimator performed slightly worse than the Ell2RSCM estimator in the Gaussian case for small $n$. It can be noticed that the performance of the LW-RSCM estimator degrades for small $n$. In the case when the samples are from a $t_{10}$-distribution, we observe that the more robust Ell1-RSCM estimator starts dominating the non-robust Ell2-RSCM estimator. Again, we note that the Ell3-RSCM estimator performed the best.

In the last synthetic simulation study, the setup is otherwise similar to the AR(1) setup in Subsection V-A, but now the sample size is held constant at $n=10$ and the degrees of 

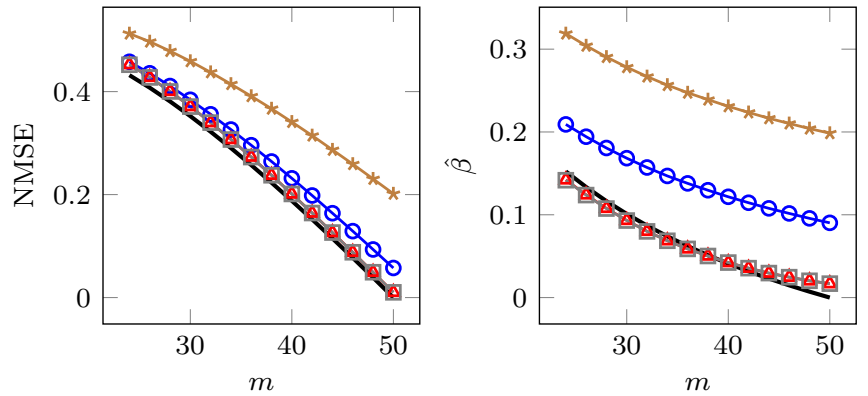

(a) Gaussian samples
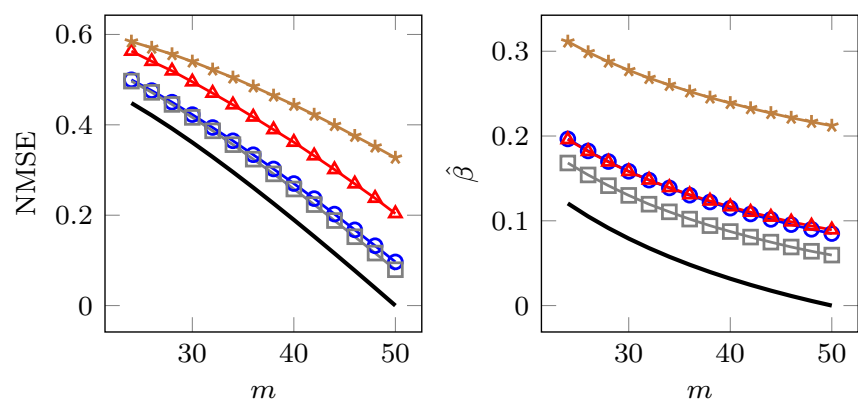

(b) $t_{10}$-distributed samples

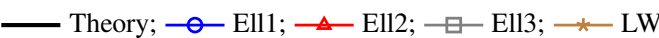

Fig. 4. The covariance matrix $\boldsymbol{\Sigma}$ has $m$ eigenvalues equal to 1 and $50-m$ eigenvalues equal to 0.01 . Here $p=50$ and $n=10$.

freedom of the $t_{\nu}$-distribution of the samples is varied from $\nu=8$ up to $\nu=1000$. The results are shown in Figure 6. One can observe that the Ell3-RSCM estimator is able to attain the lowest empirical NMSE among all of the estimators.

From these simulations, we can conclude that the Ell1RSCM estimator is better suited for heavier-tailed distributions than the Ell2-RSCM estimator, which then again works well for Gaussian or close to Gaussian distributions. The Ell3RSCM estimator is, however, able to perform the best in all of the cases. This is due to the fact that it has the freedom of choosing among two different estimates of the sphericity; and in the conducted simulations, the rule choosing the smaller estimate of the sphericity turns out to work well. In the synthetic simulations, all three proposed estimators outperformed the LW-RSCM estimator apart from the Ell2RSCM estimator in the case of $t_{7}$-distributed samples.

\section{DATA-ANALYSIS EXAMPLES}

\section{A. Regularized $Q D A$}

Suppose there are $K$ different $p$-variate populations with covariance matrix $\boldsymbol{\Sigma}_{k} \in \mathbb{S}_{++}^{p \times p}$ and a mean vector $\boldsymbol{\mu}_{k} \in \mathbb{R}^{p}$, $k=1, \ldots, K$. The problem is to classify an observation $\mathbf{x} \in$ $\mathbb{R}^{p}$ to one of the populations. We assume no knowledge of the class prior probabilities. In quadratic discriminant analysis (QDA) classification, a new observation $\mathbf{x}$ is assigned to class $\hat{k}$ by the rule

$$
\hat{k}=\underset{k \in\{1, \ldots, K\}}{\arg \min }\left(\mathbf{x}-\boldsymbol{\mu}_{k}\right)^{\top} \boldsymbol{\Sigma}_{k}^{-1}\left(\mathbf{x}-\boldsymbol{\mu}_{k}\right)+\log \left|\boldsymbol{\Sigma}_{k}\right| .
$$

Commonly, $\boldsymbol{\mu}_{k}$ and $\boldsymbol{\Sigma}_{k}$ are estimated by the sample mean vectors $\overline{\mathbf{x}}_{k}$ and the SCMs $\mathbf{S}_{k}$ computed from the training
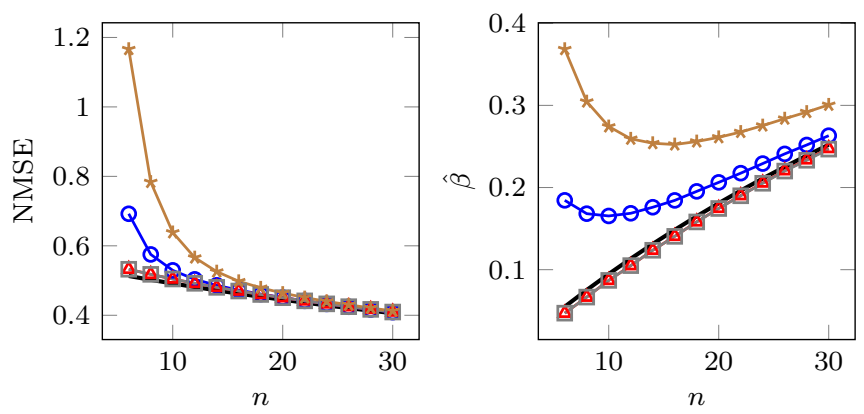

(a) Gaussian samples
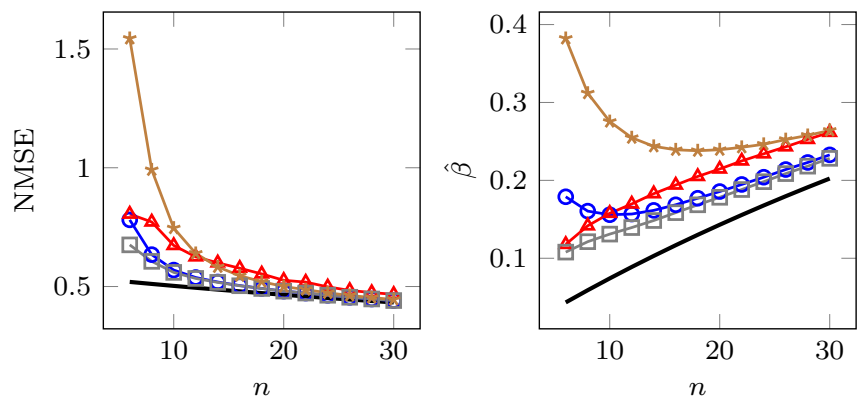

(b) $t_{10}$-distributed samples

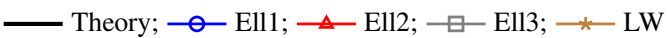

Fig. 5. The covariance matrix $\boldsymbol{\Sigma}$ has 30 eigenvalues equal to 100,40 eigenvalues equal to 1 , and 30 eigenvalues equal to $0.01(p=100)$.
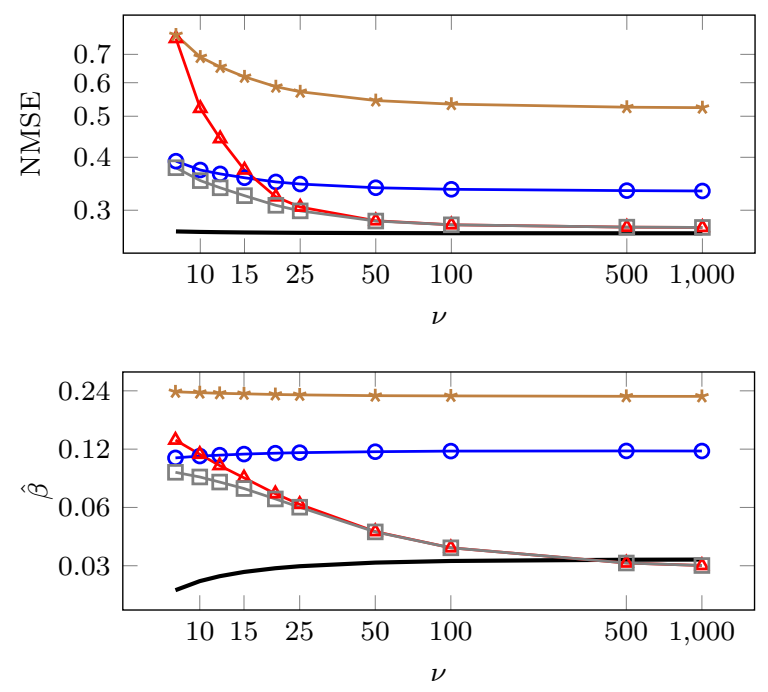

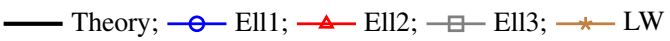

Fig. 6. The NMSE and $\hat{\beta}$ as the $t_{\nu}$-distribution changes with $\nu=8,10,12$, $15,25,50,100,500$, and 1000 . The plots are in log-log scale.

dataset $\mathbf{X}=\left(\mathbf{x}_{1} \cdots \mathbf{x}_{n}\right)$, which consists of $n_{k}$ observations from each class $k=1, \ldots, K$ and where $n=n_{1}+\cdots+n_{k}$ denotes the total sample size. In linear discriminant analysis (LDA), one assumes that the class covariance matrices are equal, so $\boldsymbol{\Sigma}=\boldsymbol{\Sigma}_{k}$ for each $k=1, \ldots, K$. Then, the unknown common covariance matrix is estimated by the pooled SCM defined as

$$
\mathbf{S}_{\text {pool }}=\sum_{k=1}^{K} \frac{n_{k}-1}{n-K} \mathbf{S}_{k}
$$


The benefit of LDA over QDA is that it can also be applied in the case when $n_{k}<p$ (for any $k$ ) as long as $n=\sum_{k} n_{k}>p$. In this case, QDA is no longer applicable since the SCM $\mathbf{S}_{k}$ is not invertible for $n_{k}<p$. LDA can be viewed as a regularized form of QDA since it decreases the variance of $\mathbf{S}_{k}$ by using the pooled SCM. LDA can often have superior performance over QDA, especially in small-sample settings.

Since the performance of LDA and QDA classification rules are highly dependent on the covariance matrix estimates, in order to reduce the misclassification rate, a popular approach is to use RSCM estimators instead of the class sample covariance matrices; see e.g., [10]. RSCMs can be applied to LDA and QDA regardless of what the available sample sizes $n_{k}$ of the classes are. Here, we use a regularized version of QDA and LDA, where we estimate the means by $\overline{\mathbf{x}}_{k}$, but use El11RSCM, Ell2-RSCM, or LW-RSCM in place of the unknown covariance matrices $\boldsymbol{\Sigma}_{k}$ in QDA and $\boldsymbol{\Sigma}$ in case of LDA. Such approach is referred to as regularized discriminant analysis (RDA) [10].

We compute the misclassification rates of LDA and QDA and different RDA methods for the phoneme dataset [27]. The original data consists of short speech frames of $32 \mathrm{msec}$ duration (512 samples with at a $16 \mathrm{kHz}$ sampling rate) and each frame represents one of the following phonemes, "aa", "ao", "dcl", "iy", or "sh" with the number of occurrencies 695, 1022, 757,1163 , and 872 , respectively. The full data set consists of 4509 speech frames spoken by 50 different male speakers. The data used for classification consists of the log-periodograms of the speech frames measured at $p=256$ distinct frequencies. The goal is to classify the spoken phonemes.

In the simulations, we randomly split the dataset into a training set and test set with the ratio 1:12. Then the sizes of the training sets were close to or smaller than the dimension $p$ as this is the regime where regularization is needed the most. The frequencies of phonemes in the training set were $53,79,58,89$, and 67 , respectively, while the remaining dataset was used as a test set. The full length of the training data was $N=\sum_{k} n_{k}=346>p=256$, and thus, the conventional LDA could be applied but the QDA could not be used as $n_{k}<p$. The misclassification rates were calculated by classifying the observations from the test set using the classification rule estimated from the training set. We report the corresponding misclassification rates based on 50 repetitions of random splits of the full data set into test sets and training sets. The boxplots of the test misclassification rates given in Figure 7 compare the conventional LDA with regularized QDA and regularized LDA. Here we also compare the performance of the Ell-RSCM estimators to an estimator that presumes Gaussianity $(\kappa=0)$ and uses the shrinkage parameter estimate $\hat{\beta}_{o}^{\text {Gau }}$ as in (23) and the estimate of the sphericity $\hat{\gamma}^{\text {Ell2 }}$ in place of the unknown $\gamma$.

Several conclusions can be drawn from Figure 7. First, the regularized LDA rules that used Ell-RSCM or LW-RSCM outperformed the LDA with a significant margin: the median test errors of the regularized LDA (resp. regularized QDA) methods based on Ell1-, Ell2-, and LW-RSCM were 9.96\%, $10.57 \%$, and $10.62 \%$. (resp. $12.86 \%, 14.36 \%$, and $15.21 \%$ ) which may be compared with the $16.8 \%$ median error rate of

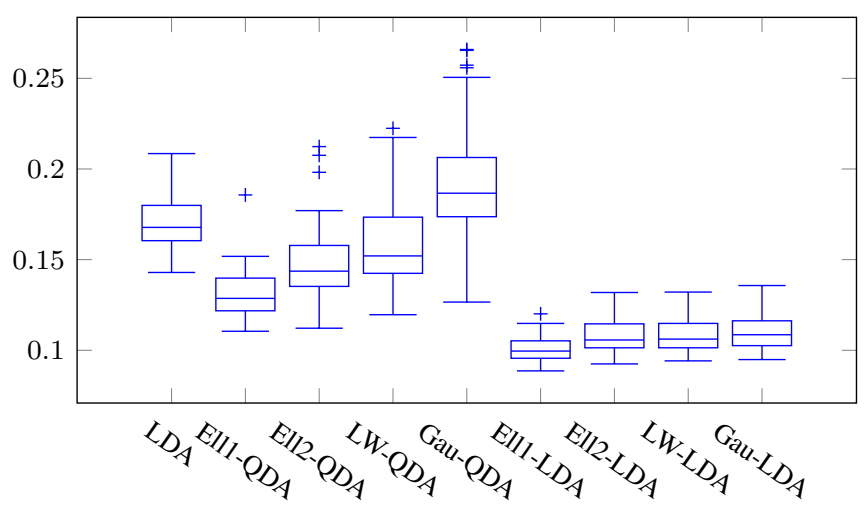

Fig. 7. Phoneme data: Box plots of the test misclassification rates of the conventional LDA compared with the regularized QDA and LDA methods based on different RSCM estimators.

the conventional LDA. Second, the overall performance of the regularized LDA methods was better than the performance of the regularized QDA methods. Third, in all cases, both Ell1RSCM and Ell2-RSCM outperformed LW-RSCM, and again, Ell1-RSCM had the best performance among all methods. Fourth, we notice that the Gau-RSCM estimator which presumes Gaussianity (and thus uses $\kappa=0$ ) is not able to perform better than the other RSCM estimators. In fact, Gau-RSCM had the worst performance among all methods when applied to the QDA rule. This illustrates the fact that the Gaussianity assumption is a poor approximation of reality for many real data analysis problems.

\section{B. Portfolio optimization}

Portfolio selection and optimization is one of the most important topics in investment theory. It is a mathematical framework wherein one seeks portfolio allocations which balance the return-risk tradeoff such that it satisfies the investor's needs. Some historical key references are [29]-[32], and [33].

Consider a portfolio consisting of $p$ assets. The objective is to find optimal portfolio weights which determine the proportion of wealth that is to be invested in each particular stock. That is, a fraction $w_{i} \in \mathbb{R}$ of the total wealth is invested in the $i$ th asset, $i=1, \ldots, p$, and the portfolio $P$ with $p$ assets is described by the portfolio weight or allocation vector $\mathbf{w} \in \mathbb{R}^{p}$ which satisfies the constraint $\mathbf{1}^{\top} \mathbf{w}=1$. The global mean variance portfolio (GMVP) aims at finding the weight vector that minimizes the portfolio variance (risk or volatility), and hence does not require specifying the mean vector. Let $\mathbf{r}_{t} \in \mathbb{R}^{p}$ denote the net returns of the $p$ assets at time $t$. The GMVP optimization problem is

$$
\underset{\mathbf{w} \in \mathbb{R}^{p}}{\operatorname{minimize}} \mathbf{w}^{\top} \mathbf{\Sigma} \mathbf{w} \quad \text { subject to } \quad \mathbf{1}^{\top} \mathbf{w}=1
$$

where 1 denotes is a $p$-vector of ones and $\boldsymbol{\Sigma}$ denotes the covariance matrix of the vector $\mathbf{r}_{t}$ of returns. The problem is straight-forward to solve and the well-known solution is

$$
\mathbf{w}_{o}=\frac{\boldsymbol{\Sigma}^{-1} \mathbf{1}}{\mathbf{1}^{\top} \boldsymbol{\Sigma}^{-1} \mathbf{1}} \text {. }
$$

Naturally, the covariances of the net returns cannot be foreseen, and hence, the covariance matrix needs to be estimated 

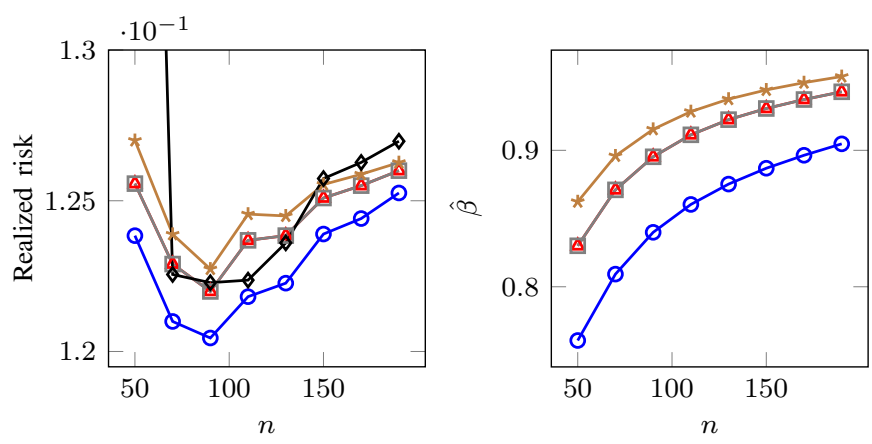

(a) HSI for Jan. 4, 2010 to Dec. 24, 2011.
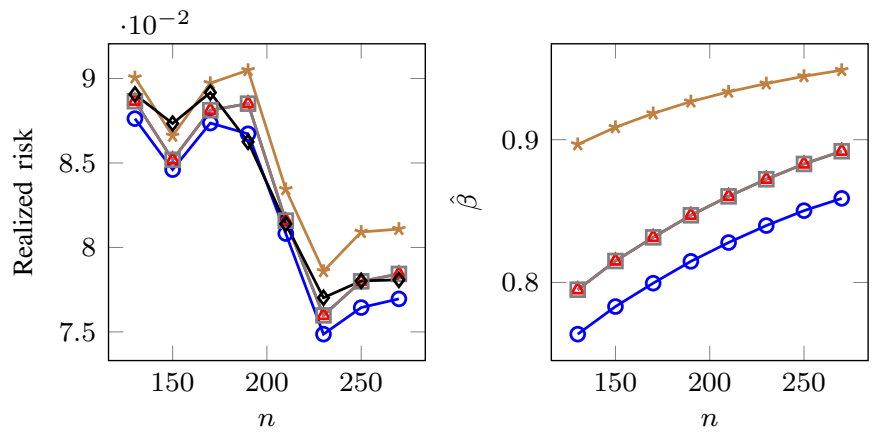

(b) HSI for Jan 1, 2016 to Dec. 27, 2017.

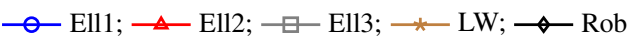

Fig. 8. Annualized realized portfolio risk and average $\hat{\beta}$ achieved out-ofsample for a portfolio consisting of $p=45$ stocks in HSI for Jan. 4, 2010 to Dec. 24, 2011 (upper panel); and $p=50$ stocks for Jan. 1, 2016 to Dec. 27, 2017 (lower panel). Both time-series contain 491 trading days. The portfolio allocations are estimated by GMVP using different RSCM estimators and different training window lengths $n$. The method of [28] that uses a robust regularized covariance estimator is also included and referred to as Rob.
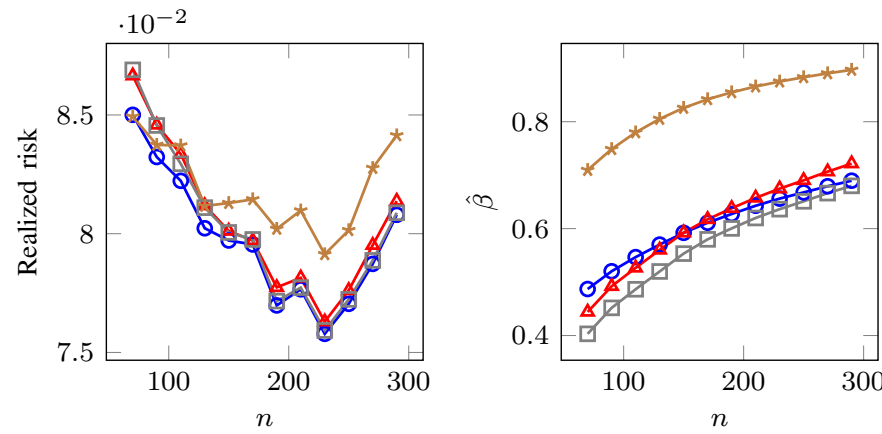

$\multimap$ Ell1; $\triangle$ Ell2; $\square-$ Ell3; —

Fig. 9. Annualized realized portfolio risk achieved out-of-sample over 583 trading days for a portfolio consisting of $p=396$ stocks in S\&P 500 index for Jan. 4, 2016 to to Apr. 27, 2018. The portfolio allocations are estimated by GMVP using different regularized SCM estimators and different training window length $n$. The right panel shows average $\hat{\beta}$ for different training windows lengths for RSCM estimators.

from the historical data. Next, we apply the proposed RSCM estimators in the GMVP optimization application.

We investigate the out-of-sample portfolio performance of different RSCM estimators. In particular, we use the divident adjusted daily closing prices downloaded from the Yahoo! Finance (http://finance.yahoo.com) database to obtain the net returns for 50 stocks that are currently included in the Hang
Seng Index (HSI) for two different time periods, from Jan. 4, 2010 to Dec. 24, 2011, and from Jan. 1, 2016 to Dec. 27, 2017 (excluding the weekends and public holidays). In both cases, the time series contain $T=491$ trading days. For the first period (2010-2011), we had full length time series for only $p=45$ stocks, whereas in the latter case we had full length time series for all stocks, so $p=50$. Our third time series contains the net returns of $p=396$ stocks from Standard and Poor's 500 (SP500) index for the time period from Jan. 4, 2016 to Apr. 27, 2018 (excluding the weekends and public holidays). In this case, the time series contains $T=583$ trading days.

At a particular day $t$, we used the previous $n$ days (i.e., from $t-n$ to $t-1$ ) as the training window to estimate the covariance matrix, and the portfolio weight vector. The obtained weight vector $\hat{\mathbf{w}}_{0}$ was then used to compute the portfolio returns for the following 20 days. Next, the window was shifted 20 trading days forward, a new weight vector was computed, and the portfolio returns for another 20 days were computed. Hence, this scenario corresponds to the case that the portfolio manager holds the assets for approximately a month (20 trading days), after which they are liquidated and new weights are computed. In this manner, we obtained $T-n$ daily returns from which the realized risk was computed as the sample standard deviation of the obtained portfolio returns. To obtain the annualized realized risk, the sample standard deviations of the daily returns were multiplied by $\sqrt{250}$. In our tests, different training window lengths $n$ were considered. Figure 8 depicts the annualized realized risks for the different RSCM estimators for both time periods of the HSI data. We also included in our study the robust GMVP weight estimator proposed in [28] that uses a robust regularized Tyler's $M$ estimator with a tuning parameter selection that is optimized for the GMVP problem. In [28], it was illustrated that their estimator outperforms a large array of regularized covariance matrix estimators both for simulated and real financial data.

As can be seen from Figure 8, for period 2010-2011, the Ell1-RSCM (and Ell3-RSCM) estimator achieved the smallest realized risk, outperforming all the other estimators for all window lengths. The robust method of [28] performed slightly better than the Ell2-RSCM estimator only for certain window lengths ( $n=70$ and $n=110$ ), but it was also the worst method for a very small window length $(n=50)$. For period 2016-2017, the differences between the estimators were not as large as in the period 2010-2011. Here we observed that for some window lengths, the Ell1- and the Ell2-RSCM estimators and the robust method of [28] had rather identical behaviour (e.g., when $n=210$ ). Overall, however, the Ell1-RSCM method was the best performing method.

Finally, we wish to point out that while Ell1-RSCM was observed to have the best performance in general, also the Ell2-RSCM estimator outperformed the LW-RSCM over the entire span of the estimation windows considered for both periods. Also, note that the optimal training window length which yielded the smallest realized risk was $n=90$ for the period 2010-2011, but much larger $(n=230)$ for the period 2016-2017. This could be explained by the fact that the stock markets were more turbulent in the first period, and hence, the realized risks were much higher. 
Figure 9 depicts the annualized realized risks for the different RSCM estimators for the time period from Jan. 4, 2016 to Apr. 27, 2018 of the SP500 data. We have excluded the method of [28] from this study as it is not well suited for very highdimensional problems because of its large computational cost due to the grid search method it uses in finding the optimal tuning parameter.

With the SP500 data, El11-RSCM achieved the smallest realized risk and outperformed the other estimators for all training window lengths $n$. The Ell2-RSCM estimator outperformed LW-RSCM when $n \geq 130$. The Ell3-RSCM estimator had similar performance as Ell2-RSCM when $n \leq 170$ and it performed similar to Ell1-RSCM for $n \geq 170$. The optimal training window length which produced the smallest realized risk was $n=230$ for all methods. Note that, the same result was achieved with the HSI data for the period 2016-2017.

\section{CONCLUSION}

This paper proposed a regularized sample covariance matrix (RSCM) estimator Ell-RSCM, which is suitable for highdimensional problems, where the data can be considered as generated from an unknown elliptically symmetric distribution. The proposed estimator is based on the estimation of the optimal shrinkage parameters which minimizes the mean squared error. The estimation of the optimal shrinkage parameters was shown to reduce to a simpler problem of estimating three statistical population parameters: the scale $\eta$, the sphericity $\gamma$, and the elliptical kurtosis $\kappa$. The paper showed alternative ways of how to estimate these parameters under the random matrix regime. In the construction of the proposed estimator Ell-RSCM, elliptical distribution theory was used in the derivation of the analytical form of the mean squared error of the SCM. The conducted synthetic simulation studies showed an advantage of using the proposed EllRSCM estimator over the widely popular Ledoit-Wolf (LWRSCM) estimator. Furthermore, we tested the performance of the proposed Ell-RSCM estimator using real data in a classification problem and in a portfolio optimization problem, wherein the proposed methods were able to outperform the benchmark methods. MATLAB codes of the proposed EllRSCM methods and codes and datasets to reproduce the results of real data-analysis examples of Section VI are available at http://users.spa.aalto.fi/esollila/regscm/.

\section{REFERENCES}

[1] O. Ledoit, M. Wolf et al., "Some hypothesis tests for the covariance matrix when the dimension is large compared to the sample size," The Annals of Statistics, vol. 30, no. 4, pp. 1081-1102, 2002.

[2] O. Ledoit and M. Wolf, "A well-conditioned estimator for largedimensional covariance matrices," Journal of Multivariate Analysis, vol. 88, pp. 365-411, 2004.

[3] Y. I. Abramovich and N. K. Spencer, "Diagonally Loaded Normalised Sample Matrix Inversion (LNSMI) for Outlier-resistant Adaptive Filtering," in IEEE International Conference on Acoustics, Speech, and Signal Processing (ICASSP), 2007.

[4] E. Ollila and D. E. Tyler, "Regularized $M$-Estimators of Scatter Matrix," IEEE Transactions on Signal Processing, vol. 62, no. 22, pp. 6059-6070, 2014.

[5] T. Zhang and A. Wiesel, "Automatic Diagonal Loading for Tyler's Robust Covariance Estimator," in IEEE Workshop on Statistical Signal Processing (SSP), 2016.
[6] Y. Sun, P. Babu, and D. P. Palomar, "Regularized Tyler's Scatter Estimator: Existence, Uniqueness, and Algorithms," IEEE Transactions on Signal Processing, vol. 62, no. 19, pp. 5143-5156, 2014.

[7] Y. Chen, A. Wiesel, Y. C. Eldar, and A. O. Hero, "Shrinkage Algorithms for MMSE Covariance Estimation," IEEE Transactions on Signal Processing, vol. 58, no. 10, pp. 5016-5029, 2010.

[8] R. Couillet and M. McKay, "Large dimensional analysis and optimization of robust shrinkage covariance matrix estimators," Journal of Multivariate Analysis, vol. 131, pp. 99-120, 2014.

[9] F. Pascal, Y. Chitour, and Y. Quek, "Generalized Robust Shrinkage Estimator and Its Application to STAP Detection Problem," IEEE Transactions on Signal Processing, vol. 62, no. 21, pp. 5640-5651, 2014.

[10] J. H. Friedman, "Regularized Discriminant Analysis," Journal of the American Statistical Association, vol. 84, no. 405, pp. 165-175, 1989.

[11] B. D. Carlson, "Covariance Matrix Estimation Errors and Diagonal Loading in Adaptive Arrays," IEEE Transactions on Aerospace and Electronic Systems, vol. 24, no. 4, pp. 397-401, 1988.

[12] Y. Abramovich, "Controlled Method for Adaptive Optimization of Filters Using the Criterion of Maximum Signal-to-Noise Ratio," Radio Eng. Elect. Phys., vol. 26, no. 3, pp. 87-95, 1981.

[13] N. Auguin, D. Morales-Jimenez, M. McKay, and R. Couillet, "Robust Shrinkage $M$-Estimators of Large Covariance Matrices," in IEEE Workshop on Statistical Signal Processing (SSP), 2016.

[14] R. J. Muirhead, Aspects of Multivariate Statistical Theory. Wiley, 1982.

[15] K.-T. Fang, S. Kotz, and K.-W. Ng, Symmetric Multivariate and Related Distributions. Chapman and Hall/CRC, 1990.

[16] E. Ollila, D. E. Tyler, V. Koivunen, and H. V. Poor, "Complex Elliptically Symmetric Distributions: Survey, New Results and Applications," IEEE Transactions on Signal Processing, vol. 60, no. 11, pp. 5597-5625, 2012.

[17] J. R. Magnus and H. Neudecker, Matrix Differential Calculus with Applications in Statistics and Econometrics, 2nd ed. Wiley, 1999.

[18] M. S. Srivastava, "Some Tests Concerning the Covariance Matrix in High Dimensional Data," Journal of the Japan Statistical Society, vol. 35, no. 2, pp. 251-272, 2005.

[19] D. E. Tyler, "Radial estimates and the test for sphericity," Biometrika, vol. 69 , no. 2 , pp. 429-436, 1982.

[20] E. Ollila, "Optimal High-Dimensional Shrinkage Covariance Estimation for Elliptical Distributions," in 25th European Signal Processing Conference (EUSIPCO), 2017

[21] D. N. Joanes and C. A. Gill, "Comparing Measures of Sample Skewness and Kurtosis," Journal of the Royal Statistical Society: Series D (The Statistician), vol. 47, no. 1, pp. 183-189, 1998.

[22] P. M. Bentler and M. Berkane, "Greatest lower bound to the elliptical theory kurtosis parameter," Biometrika, vol. 73, no. 1, pp. 240-241, 1986.

[23] M. N. Tabassum and E. Ollila, "Compressive Regularized Discriminant Analysis of High-Dimensional Data With Applications to Microarray Studies," IEEE International Conference on Acoustics, Speech, and Signal Processing (ICASSP), pp. 4204-4208, 2018.

[24] B. Brown, "Statistical Uses of the Spatial Median," Journal of the Royal Statistical Society. Series B (Methodological), vol. 45, no. 1, pp. 25-30, 1983.

[25] C. Croux and E. Ollila, "Sign and Rank Covariance Matrices: Statistical Properties and Application to Principal Components Analysis," Statistical Data Analysis Based on the L1-norm and Related Methods, pp. 257-269, 2002

[26] A. F. Magyar and D. E. Tyler, "The asymptotic inadmissibility of the spatial sign covariance matrix for elliptically symmetric distributions," Biometrika, vol. 101, no. 3, pp. 673-688, 2014.

[27] T. Hastie, R. Tibshirani, and J. Friedman, The elements of statistical learning. New York: Springer, 2001.

[28] L. Yang, R. Couillet, and M. R. McKay, "A robust statistics approach to minimum variance portfolio optimization," IEEE Transactions on Signal Processing, vol. 63, no. 24, pp. 6684-6697, 2015.

[29] H. Markowitz, "Portfolio Selection," The Journal of Finance, vol. 7, no. 1, pp. 77-91, 1952.

[30] — Portfolio Selection, Efficient Diversification of Investments. Wiley, 1959.

[31] J. Tobin, "Liquidity Preference as Behavior Towards Risk," The Review of Economic Studies, vol. 25, no. 2, pp. 65-86, 1958.

[32] W. Sharpe, "Capital Asset Prices: A Theory of Market Equilibrium Under Conditions of Risk," The Journal of Finance, vol. 19, no. 3 , pp. 425-442, 1964.

[33] J. Lintner, "The Valuation of Risk Assets and the Selection of Risky Investments in Stock Portfolios and Capital Budgets," The Review of Economics and Statistics, vol. 47, no. 1, pp. 13-37, 1965. 